\title{
Structure of Basic Lie Superalgebras and of their Affine Extensions
}

\author{
L. Frappat ${ }^{1,3}$, A. Sciarrino ${ }^{2}$, and P. Sorba ${ }^{1}$ \\ ${ }^{1}$ L.A.P.P., B.P. 909, F-74019 Annecy-le-Vieux, France \\ ${ }^{2}$ Dipartimento di Fisica, I-80125 Napoli, Italy, and I.N.F.N., Sezione di Napoli, Italy \\ ${ }^{3}$ Université de Savoie, F-74000 Annecy, France
}

\begin{abstract}
We generalize to the case of superalgebras several properties of simple Lie algebras involving the use of Dynkin diagrams. If to a simple Lie algebra can be associated one Dynkin diagram, it is a finite set of nonequivalent ones which can be constructed for a basic superalgebra (or B.S.A.). The knowledge of these diagrams, which can be obtained for each B.S.A. in a systematic way, allows us to deduce the regular subsuperalgebras of a B.S.A. The symmetries of the Dynkin diagrams are related to outer automorphisms of B.S.A. and lead to some singular subsuperalgebras. Finally we consider the extended Dynkin diagrams in order to classify the affine B.S.A. and use their symmetries to construct the twisted basic superalgebras.
\end{abstract}

\section{Introduction}

In his classification of simple Lie superalgebras, Kac [1-3] distinguishes two general families: the classical Lie superalgebras in which the representation of the even subalgebra on the odd part is completely reducible and the Cartan type superalgebras in which such a property is not valid. Among the classical superalgebras, one naturally separates the "strange" series $P(n)$ and $Q(n)$ from the basic or contragredient superalgebras which include the $A(m, n)$ unitary series, the $B(m, n), C(n+1)$, and $D(m, n)$ orthosymplectic series and the exceptional superalgebras $F(4)$ and $G(3)$ as well as $D(2,1 ; \alpha)$ - these last ones being a deformation of $D(2,1)$. These basic superalgebras - up to now denoted by B.S.A. are in some extent very close to the usual simple Lie algebras. For example, they can be studied with the help of Cartan matrices and Dynkin diagrams. However a fundamental difference with the Lie algebras occurs at this level because of the unavoidable presence in the simple root systems of odd - or fermionic - roots together with even - or bosonic - ones. Indeed for each simple Lie algebra $\mathscr{A}$, there is only one simple root system, up to a transformation of the Weyl group $W(\mathscr{A})$. In a B.S.A. several unequivalent simple root systems, that is systems which cannot be related one to each other by a Weyl transformation, can be in general defined, 
leading to different Cartan matrices and Dynkin diagrams. Let us make precise that the Weyl group in a B.S.A. is generated by the reflections associated with the bosonic roots, and therefore is isomorphic to the Weyl group of the even part. This special feature [1] of B.S.A. has been considered in detail in [4-6], and a practical method to construct all the simple root systems of a B.S.A. from a given one has been described in [5].

The complete knowledge of the Dynkin diagram can be exploited in different directions. First, by extending the Dynkin diagram of a B.S.A. one can repeat the Dynkin method for obtaining the regular subalgebras of a simple algebra to the case of a B.S.A., and thus construct the regular subsuperalgebras of B.S.A. Secondly, as for simple Lie algebras, the symmetry of the Dynkin diagram must be related to the outer automorphisms of B.S.A. Such symmetries can in particular be used to construct some non-regular - or singular - subsuperalgebras. Moreover, the extended diagrams can be considered in order to classify the affine untwisted B.S.A. Symmetries of the Dynkin diagrams could then be studied to obtain the twisted affine B.S.A. as well as special inclusions among them.

The paper is organized as follows. Section 2 is devoted to the study of the simple root systems of a B.S.A. $\mathscr{G}$, the definition of the Cartan matrix and the classification of the Dynkin diagrams associated to $\mathscr{G}$. With these tools at hand, we can construct in Sect. 3 the regular sub(super)algebras of a B.S.A., and in Sect. 4 make a correspondance between the symmetries of the Dynkin diagram of a B.S.A. and its outer automorphisms. The case of affine B.S.A. is considered in Sect. 5: symmetries of the Dynkin diagrams are then directly used to construct in an explicit way the twisted B.S.A. We conclude by mentioning some possible developments and physical applications.

Although the properties on superalgebras contained in Sects. 2 and 3 do not constitute new results, it has seemed to us useful to present them in a synthetic way, mainly for the two following reasons. First we want to show as far as possible how properties of simple algebras can be extended to superalgebras. Such a program becomes much easier owing to the practical tool proposed in [5], i.e. the use of Weyl transformations associated to zero length roots allowing us to construct from a given one all the Dynkin diagrams of a superalgebra. Secondly, introducing in some detail such basic properties makes more clear our diagrammatic study of outer automorphisms for basic superalgebras in Sect. 4, as well as the construction of twisted affine superalgebras via outer automorphisms of extended Dynkin diagrams in Sect. 5.

\section{Cartan Matrix and Dynkin Diagrams for B.S.A.}

\subsection{Notations and First Properties}

Let us start with a brief reminder about root systems of basic superalgebras. The Lie bracket in a Lie superalgebra $\mathscr{G}=\mathscr{G}_{0}+\mathscr{G}_{1}$ is defined by the equality:

$$
[a, b]=a b-(-1)^{(\operatorname{deg} a)(\operatorname{deg} b)} b a \text { for all } a, b \in \mathscr{G},
$$

the degree being 0 for elements of the subalgebra $\mathscr{G}_{0}$ and 1 for elements of the $\mathscr{G}_{0^{-}}$ representation $\mathscr{G}_{1}$. Moreover, one has globally:

$$
\left[\mathscr{G}_{0}, \mathscr{G}_{0}\right] \subset \mathscr{G}_{0} \quad\left[\mathscr{G}_{0}, \mathscr{G}_{1}\right] \subset \mathscr{G}_{1} \quad\left[\mathscr{G}_{1}, \mathscr{G}_{1}\right] \subset \mathscr{G}_{0} .
$$


Let $H$ be a Cartan subalgebra of $\mathscr{G}_{0}$. A root $\alpha$ of $\mathscr{G}(\alpha \neq 0)$ will be an element $\alpha \in H^{*}$, the dual of $H$, such that:

$$
\mathscr{G}_{\alpha}=\{a \in \mathscr{G} \mid[h, a]=\alpha(h) \cdot a, h \in H\} \neq 0 .
$$

A root $\alpha$ is called even or bosonic (respectively odd or fermionic) if $\mathscr{G}_{0} \cap \mathscr{G}_{\alpha} \neq 0$ (respectively $\mathscr{G}_{1} \cap \mathscr{G}_{\alpha} \neq 0$ ). A contragredient or basic superalgebra is a superalgebra such that $\operatorname{dim} \mathscr{G}_{\alpha}=\operatorname{dim} \mathscr{G}_{-\alpha}$ for any root $\alpha$ of $\mathscr{G}$. The Killing form on $\mathscr{G}$ is the bilinear form:

$$
(a, b)=S \operatorname{Tr}(\operatorname{Ad} a)(\operatorname{Ad} b) \text {. }
$$

Note at this point that, if $\operatorname{dim} \mathscr{G}_{0}=m$ and $\operatorname{dim} \mathscr{G}_{1}=n, \operatorname{Ad}(a)$ for any $a \in \mathscr{G}$ can be seen as a $(m+n) \times(m+n)$ matrix $M$

$$
M=\left(\begin{array}{ll}
A & B \\
C & D
\end{array}\right),
$$

where $A$ (respectively $D$ ) is a $m \times m$ (respectively $n \times n)$ matrix, and the supertrace of $M$ acting on the $Z_{2}$ graded space $\mathscr{G}$ is then defined as:

$$
S \operatorname{Tr} M=\operatorname{Tr} A-\operatorname{Tr} D .
$$

Now let us rapidly introduce the different families of basic superalgebras and define their corresponding root system: we will denote by $\Delta_{0}$ (respectively $\Delta_{1}$ ) the set of even (respectively odd) roots.

- The unitary series $A(m, m)$ or $S l(m+11 n+1)$ :

The bosonic part is $\mathscr{G}_{0}=S l(m+1) \oplus S l(n+1) \oplus U(1)$ if $m \neq n$ (the $U(1)$ part being absent if $m=n)$, and $\mathscr{G}_{1}$ reduces with respect to the $S l(m+1) \oplus \operatorname{Sl}(n+1)$ group as the representation $(m+1, \overline{n+1})+(\overline{m+1}, n+1)$.

The roots can be expressed in terms of $\varepsilon_{1}, \ldots, \varepsilon_{m+1}, \delta_{1}, \ldots, \delta_{n+1}$ as:

$$
\Delta_{0}=\left\{\varepsilon_{i}-\varepsilon_{j} ; \delta_{l}-\delta_{j}\right\} \quad \Delta_{1}=\left\{ \pm\left(\varepsilon_{i}-\delta_{j}\right)\right\} .
$$

- The orthosymplectic series $\operatorname{OSp}(m \mid 2 n)$ :

The bosonic part is a non-compact form of $O(m) \oplus S p(2 n)$ and $\mathscr{G}_{1}$ reduces for $m \neq 2$ to the $(m, 2 n) \mathscr{G}_{0}$-representation.

The roots expressed in terms of $\varepsilon_{1}, \ldots, \varepsilon_{m}, \delta_{1}, \ldots, \delta_{n}$ are:

for $B(m, n)$ or $O S p(2 m+1 \mid 2 n)$ with $m \neq 0$

$$
\begin{gathered}
\Delta_{0}=\left\{ \pm \varepsilon_{i} \pm \varepsilon_{j} ; \pm \varepsilon_{i} ; \pm \delta_{i} \pm \delta_{j} ; \pm 2 \delta_{i}\right\} \quad(i \neq j) \\
\Delta_{1}=\left\{ \pm \delta_{i} ; \pm \varepsilon_{i} \pm \delta_{i}\right\},
\end{gathered}
$$

for $B(0, n)$ or $\operatorname{OSp}(1 \mid 2 n)$

$$
\Delta_{0}=\left\{ \pm \delta_{i} \pm \delta_{j} ; \pm 2 \delta_{i}\right\} \quad(i \neq j) \quad \Delta_{1}=\left\{ \pm \delta_{i}\right\},
$$

for $D(m, n)$ or $O S p(2 m \mid 2 n)$ with $m \neq 1$

$$
\begin{gathered}
\Delta_{0}=\left\{ \pm \varepsilon_{i} \pm \varepsilon_{j} ; \pm \delta_{i} \pm \delta_{j} ; \pm 2 \delta_{i}\right\} \quad(i \neq j) \\
\Delta_{1}=\left\{ \pm \varepsilon_{i} \pm \delta_{j}\right\},
\end{gathered}
$$


while for $C(n+1)$ of $O S p(2 \mid 2 n)$ the odd part is $\mathscr{G}_{1}$ twice the fundamental $(2 n)$ representation of $S p(2 n)$, and the roots in terms of $\varepsilon, \delta_{1}, \ldots, \delta_{n}$ are:

$$
\begin{gathered}
\Delta_{0}=\left\{ \pm \delta_{i} \pm \delta_{j} ; \pm 2 \delta_{i}\right\} \quad(i \neq j) \\
\Delta_{1}=\left\{ \pm \varepsilon \pm \delta_{i}\right\} .
\end{gathered}
$$

- The exceptional superalgebras:

-- $D(2,1 ; \alpha)$ (with $\alpha \neq 0,-1)$ which are deformations of $D(2,1)$, corresponding actually to the case $\alpha=1$. The bosonic part is a non-compact form of $S U(2) \oplus S U(2) \oplus S U(2)$ and the fermionic one $\mathscr{G}_{1}$ is the 8-dimensional $(2,2,2)$ $\mathscr{G}_{0}$-representation. In terms of $\varepsilon_{1}, \varepsilon_{2}, \varepsilon_{3}$ the roots are:

$$
\Delta_{0}=\left\{ \pm 2 \varepsilon_{i}\right\} \quad \Delta_{1}=\left\{ \pm \varepsilon_{1} \pm \varepsilon_{2} \pm \varepsilon_{3}\right\} \text {. }
$$

$F(4)$ the $\mathscr{G}_{0}$ part of which is a non-compact form of $S U(2) \oplus O(7)$ and the $\mathscr{G}_{1}$ part the $(2,8) \mathscr{G}_{0}$-representation. Note that $D(2,1 ; \alpha)$ and $F(4)$ share the property to have for $\mathscr{G}_{1}$ spinorial representations of their $\mathscr{G}_{0}$ part. In terms of $\delta$ and $\varepsilon_{1}, \varepsilon_{2}, \varepsilon_{3}$ the roots are:

$$
\Delta_{0}=\left\{ \pm \delta ; \pm \varepsilon_{i} \pm \varepsilon_{j} ; \pm \varepsilon_{i}\right\} \quad \Delta_{1}=\left\{\frac{1}{2}\left( \pm \varepsilon_{1} \pm \varepsilon_{2} \pm \varepsilon_{3} \pm \delta\right)\right\} .
$$

- $G(3)$ with a non-compact form of $S U(2) \oplus \mathscr{G}_{2}$, if $\mathscr{G}_{2}$ is the Lie algebra of the exceptional Lie group $G_{2}$, for the $\mathscr{G}_{0}$ part and the $(2,7) \mathscr{G}_{0}$-representation for the $\mathscr{G}_{1}$ part. In terms of $\delta$ and $\varepsilon_{1}, \varepsilon_{2}, \varepsilon_{3}$ with $\varepsilon_{1}+\varepsilon_{2}+\varepsilon_{3}=0$ the roots are:

$$
\Delta_{0}=\left\{ \pm 2 \delta ; \varepsilon_{i}-\varepsilon_{j} ; \pm \varepsilon_{i}\right\} \quad \Delta_{1}=\left\{ \pm \delta ; \pm \varepsilon_{i} \pm \delta\right\} \text {. }
$$

This classification can be closed by a few remarks. One can find B.S.A. such that the representation of $\mathscr{G}_{0}$ on $\mathscr{G}_{1}$ is irreducible, and $\mathscr{G}_{0}$ is semi-simple: this is the case for the superalgebras $B(m, n), D(m, n), F(4), G(3)$ and $D(2,1 ; \alpha)$ which are called superalgebras of type II. The other ones, i.e. $A(m, n)$ and $C(n+1)$, admit a unique consistent $Z_{2}$ gradation of the form $\mathscr{G}=\mathscr{G}_{-1} \oplus \mathscr{G}_{0} \oplus \mathscr{G}_{1}$ (i.e. $\left[\mathscr{G}_{i}, \mathscr{G}_{j}\right] \subset \mathscr{G}_{i+j}(\bmod 2)$ with $\left.i, j=-1,0,1\right)$ with the $\mathscr{G}_{0}$-modules $\mathscr{G}_{-1}$ and $\mathscr{G}_{1}$ irreducible. They are called superalgebras of type $I$.

Among the fermionic roots, one can notice that there exist roots $\delta$ such that $2 \delta$ is a bosonic root. Such a root will be associated with the B.S.A. $O S p(1 \mid 2)$. The other fermionic roots will be associated with the B.S.A. Sl(1|1).

\subsection{Cartan Matrix}

In a contragredient Lie superalgebra $\mathscr{G}$ of rank $r$, it is always possible to define a $r \times r$ Cartan matrix $A=\left(a_{i j}\right)$ associated to a set of simple roots $\left(\alpha_{1}, \ldots, \alpha_{r}\right)$ with the following conditions:

$$
\begin{aligned}
{\left[h_{i}, e_{ \pm \alpha}\right] } & = \pm a_{i j} e_{ \pm \alpha_{j}}, \\
{\left[e_{\alpha_{2}}, e_{-\alpha_{j}}\right] } & =\delta_{i j} h_{i}, \\
{\left[h_{i}, h_{j}\right] } & =0,
\end{aligned}
$$

the $h_{1}, \ldots, h_{n}$ generating the corresponding Cartan subalgebra $H$.

One defines, using Eq.(2.4), a non-degenerate bilinear form on $H^{*}$ by:

$$
\left(\alpha_{i}, \alpha_{j}\right) \equiv\left(h_{i}, h_{j}\right)
$$


such a form being invariant under the Weyl group of $\mathscr{G}$, generated by the reflections of the bosonic roots. The Cartan matrix can be chosen symmetric [6] and defined as follows:

$$
a_{i j}=\left(\alpha_{i}, \alpha_{j}\right)
$$

once the normalization on the Cartan generators is fixed, or equivalently in the root lattice.

Note that such a Cartan matrix can be obtained from the Kac-Cartan matrix defined in [2] by multiplying this last one on the left by the following diagonal matrix (where $\mathbf{1}_{m}$ denotes the $m \times m$ identity matrix):

$$
\begin{aligned}
& \left(\begin{array}{ccc}
\mathbf{1}_{m} & 0 & 0 \\
0 & 1 & 0 \\
0 & 0 & -\mathbf{1}_{n}
\end{array}\right) \quad \text { for } \operatorname{Sl}(m+1 \mid n+1), \\
& \left(\begin{array}{cc}
\mathbf{1}_{n} & 0 \\
0 & -\mathbf{1}_{m}
\end{array}\right) \quad \text { for } \operatorname{OSp}(2 m \mid 2 n) \quad(m \neq 1), \\
& \left(\begin{array}{ccc}
\mathbf{1}_{n} & 0 & 0 \\
0 & -\mathbf{1}_{m-1} & 0 \\
0 & 0 & -2
\end{array}\right) \quad \text { for } \operatorname{OSp}(2 m+1 \mid 2 n) \quad(m \neq 0), \\
& \left(\begin{array}{ccc}
1 & 0 & 0 \\
0 & 1_{n-1} & 0 \\
0 & 0 & -1 / 2
\end{array}\right) \quad \text { for } \operatorname{OSp}(2 \mid 2 n) \\
& \left(\begin{array}{ccc}
1 & 0 & 0 \\
0 & -1 & 0 \\
0 & 0 & -1 / 3
\end{array}\right) \quad \text { for } G(3) \\
& \left(\begin{array}{cccc}
1 & 0 & 0 & 0 \\
0 & -1 & 0 & 0 \\
0 & 0 & -1 / 2 & 0 \\
0 & 0 & 0 & -1 / 2
\end{array}\right) \quad \text { for } F(4) \\
& \frac{-1}{1+\alpha}\left(\begin{array}{ccc}
\frac{2+\alpha}{2} & 2+\alpha & 1 / 2 \\
\frac{4+\alpha}{2} & 3 & 3 / 2 \\
\frac{2+\alpha}{2} & 1 & -\frac{2+\alpha}{2 \alpha}
\end{array}\right] \quad \text { for } D(2,1 ; \alpha)
\end{aligned}
$$

Now we shall associate to each simple root system of $\mathscr{G}$ a Dynkin diagram according to the following rules:

i) To each simple bosonic root we associate a white dot $\bigcirc$, to each simple fermionic one $\alpha_{i}$ a black dot $\bullet$ if $a_{i i} \neq 0$ (i.e. $2 \alpha_{i} \in \Delta_{0}$ ) and a grey dot $\bigcirc$ if $a_{i i}=0$. 
ii) The $i^{\text {th }}$ and $j^{\text {th }}$ dot will be joined by $\eta_{i j}$ lines with:

$$
\begin{array}{ll}
\eta_{i j}=2 \frac{\left|a_{i j}\right|}{\min \left(\left|a_{i i}\right|,\left|a_{j j}\right|\right)} & \text { if } a_{i i} \cdot a_{j j} \neq 0, \\
\eta_{i j}=2 \frac{\left|a_{i j}\right|}{\min _{a_{k k} \neq 0}\left|a_{k k}\right|} & \text { if } a_{i i} \neq 0, a_{j j}=0, \\
\eta_{i j}=\left|a_{i j}\right| & \text { if } a_{i i}=a_{j j}=0 .
\end{array}
$$

iii) We add an arrow on the lines connecting the $i^{\text {th }}$ and $j^{\text {th }}$ dot when $\eta_{i j}>1$, pointing from $i$ to $j$ if $a_{i i} \cdot a_{j j} \neq 0$ and $\left|a_{j j}\right|$ or if $a_{i i}=0, a_{j j} \neq 0,\left|a_{j j}\right|<2$ and pointing from $j$ to $i$ if $a_{i i}=0, a_{j j} \neq 0,\left|a_{j j}\right|>2$.

Let us emphasize at this point that to a given superalgebra $\mathscr{G}$ will not in general be associated only one system of simple roots up to a transformation of the Weyl group $W(\mathscr{G})$, and therefore not only one Dynkin diagram. This will be discussed in more detail in the subsequent paragraph. We want for the moment to illustrate the above rules by constructing for each kind of B.S.A. a particular Dynkin diagram related to a special simple root system. We will call such a system a "distinguished" one, its characteristic being that it contains the smallest number of fermionic roots. Note that the labels appearing above each dot are the coefficients of the decomposition of the highest root with respect to the simple roots.

- $A(m, n)$

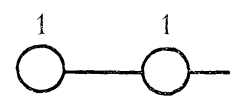

$\varepsilon_{1}-\varepsilon_{2} \varepsilon_{2}-\varepsilon_{3}$

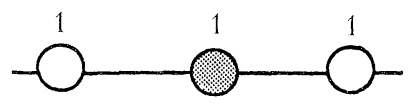

$\varepsilon_{m}-\varepsilon_{m+1} \quad \varepsilon_{m+1}-\delta_{1} \quad \delta_{1}-\delta_{2}$

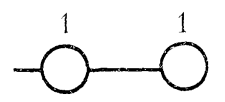

$\delta_{n-1}-\delta_{n} \delta_{n}-\delta_{n+1}$

with the normalization

$\left(\varepsilon_{i}, \varepsilon_{j}\right)=\delta_{i j}(i, j=1, \ldots, m+1), \quad\left(\delta_{k}, \delta_{l}\right)=-\delta_{k l}(k, l=1, \ldots, n+1), \quad\left(\varepsilon_{i}, \delta_{k}\right)=0$

and the corresponding Cartan matrix

$$
\left[\begin{array}{ccccccc}
2 & -1 & & & & & \\
-1 & \ddots & \ddots & & & & \\
& \ddots & 2 & -1 & & & \\
& & -1 & 0 & 1 & & \\
& & & 1 & -2 & \ddots & \\
& & & & \ddots & \ddots & 1 \\
& & & & & 1 & -2
\end{array}\right] .
$$

- $B(m, n)(m>0)$
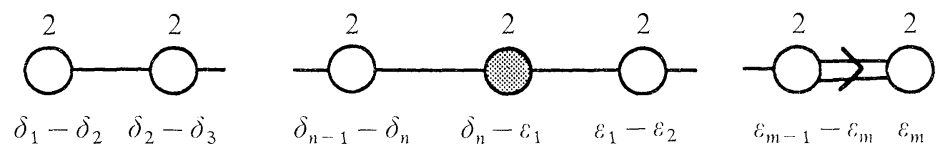
with the normalization

$$
\left(\varepsilon_{i}, \varepsilon_{j}\right)=-\delta_{i j}(i, j=1, \ldots, m), \quad\left(\delta_{k}, \delta_{l}\right)=\delta_{k l}(k, l=1, \ldots, n), \quad\left(\varepsilon_{i}, \delta_{k}\right)=0
$$

and the corresponding Cartan matrix

$$
\left[\begin{array}{ccccccc}
2 & -1 & & & & & \\
-1 & \ddots & \ddots & & & & \\
& \ddots & 2 & -1 & & & \\
& & -1 & 0 & 1 & & \\
& & & 1 & -2 & \ddots & \\
& & & & \ddots & \ddots & 1 \\
& & & & & 1 & -1
\end{array}\right] .
$$

- $B(0, n)$
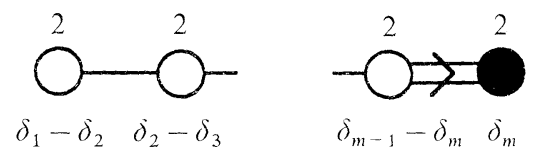

with the normalization

$$
\left(\delta_{k}, \delta_{l}\right)=\delta_{k l}(k, l=1, \ldots, n)
$$

and the corresponding Cartan matrix

$$
\left(\begin{array}{cccc}
2 & -1 & & \\
-1 & \ddots & \ddots & \\
& \ddots & 2 & -1 \\
& & -1 & 1
\end{array}\right)
$$

- $C(n+1)$
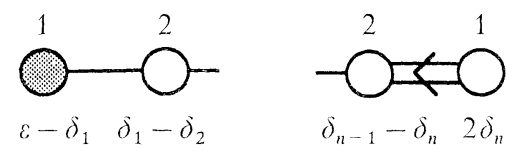

with the normalization

$$
(\varepsilon, \varepsilon)=1, \quad\left(\delta_{k}, \delta_{l}\right)=-\delta_{k l}(k, l=1, \ldots, n+1), \quad\left(\varepsilon, \delta_{k}\right)=0
$$

and the corresponding Cartan matrix

$$
\left[\begin{array}{cccccccc}
0 & -1 & & & & & & \\
-1 & \ddots & \ddots & & & & & \\
& \ddots & 2 & -1 & & & & \\
& & -1 & 0 & 1 & & & \\
& & & 1 & -2 & \ddots & & \\
& & & & \ddots & \ddots & 1 & \\
& & & & & 1 & -2 & 2 \\
& & & & & & 2 & -4
\end{array}\right]
$$


- $D(m, n)$

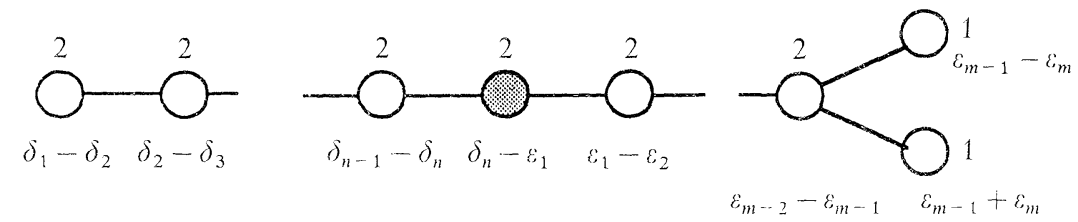

with the normalization

$$
\left(\varepsilon_{i}, \varepsilon_{j}\right)=-\delta_{i j}(i, j=1, \ldots, m), \quad\left(\delta_{k}, \delta_{l}\right)=\delta_{k l}(k, l=1, \ldots, n), \quad\left(\varepsilon_{i}, \delta_{k}\right)=0
$$

and the corresponding Cartan matrix

$$
\left[\begin{array}{cccccccc}
2 & -1 & & & & & & \\
-1 & \ddots & \ddots & & & & & \\
& \ddots & 2 & -1 & & & & \\
& & -1 & 0 & 1 & & & \\
& & & 1 & -2 & \ddots & & \\
& & & & \ddots & \ddots & 1 & 1 \\
& & & & & 1 & -2 & 0 \\
& & & & & 1 & 0 & -2
\end{array}\right]
$$

- $G(3)$

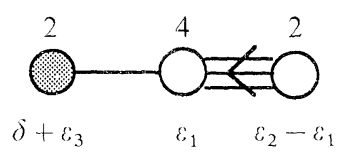

with the normalization

$$
\left(\varepsilon_{i}, \varepsilon_{j}\right)=-3 \dot{\delta}_{i j}+1(i, j=1,2,3), \quad(\delta, \delta)=2, \quad\left(\varepsilon_{i}, \delta\right)=0
$$

and the corresponding Cartan matrix

- $F(4)$

$$
\left(\begin{array}{ccc}
0 & 1 & 0 \\
1 & -2 & 3 \\
0 & 3 & -6
\end{array}\right)
$$

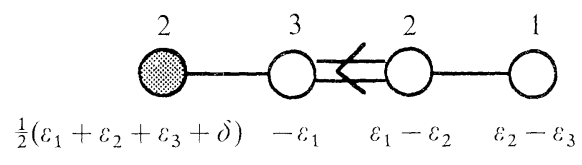

with the normalization

$$
\left(\varepsilon_{i}, \varepsilon_{j}\right)=-2 \delta_{i j}(i, j=1,2,3), \quad(\delta, \delta)=6, \quad\left(\varepsilon_{i}, \delta\right)=0
$$


and the corresponding Cartan matrix

$$
\left(\begin{array}{cccc}
0 & 1 & 0 & 0 \\
1 & -2 & 2 & 0 \\
0 & 2 & -4 & 2 \\
0 & 0 & 2 & -4
\end{array}\right)
$$

- $D(2,1 ; \alpha)$

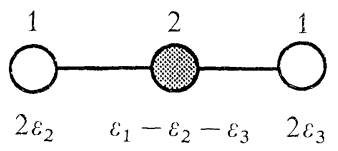

with the normalization

$$
\left(\varepsilon_{1}, \varepsilon_{1}\right)=-(1+\alpha) / 2, \quad\left(\varepsilon_{2}, \varepsilon_{2}\right)=1 / 2, \quad\left(\varepsilon_{3}, \varepsilon_{3}\right)=\alpha / 2, \quad\left(\varepsilon_{i}, \varepsilon_{j}\right)=0(i \neq j)
$$

and the corresponding Cartan matrix

$$
\left(\begin{array}{ccc}
2 & -1 & 0 \\
-1 & 0 & -\alpha \\
0 & -\alpha & 2 \alpha
\end{array}\right)
$$

\subsection{Simple Root System of a Superalgebra}

In this subsection we describe a very practical way [5] to obtain all the simple root systems - or equivalently all the Dynkin diagrams - of a given B.S.A. once given any one of them.

Let us recall that the Weyl group $W(\mathscr{G})$ of a superalgebra $\mathscr{G}$ is generated by the Weyl reflections relative to the bosonic roots - such reflections read:

$$
\omega_{\alpha}(\beta)=\beta-2 \frac{(\alpha, \beta)}{(\alpha, \alpha)} \alpha
$$

with $\alpha \in \Delta_{0}(\mathscr{G})$ and $\beta \in \Delta_{0}(\mathscr{G}) \cup \Delta_{1}(\mathscr{G})$. Under a transformation of $W(\mathscr{G})$, a simple root system will be transformed into an equivalent one, with the same Dynkin diagram.

Now let us, following [5], extend $W(\mathscr{G})$ to a larger group by adding the following transformations associated to fermionic roots $\alpha \in \Delta_{1}$ :

$$
\begin{aligned}
& (\alpha, \alpha) \neq 0 \quad \omega_{\alpha}(\beta)=\beta-2 \frac{(\alpha, \beta)}{(\alpha, \alpha)} \alpha \\
& (\alpha, \alpha)=0 \quad \omega_{\alpha}(\beta)=\beta+\alpha \quad \text { if }(\alpha, \beta) \neq 0 \\
& =\beta \quad \text { if }(\alpha, \beta)=0 \\
& \omega_{\alpha}(\alpha)=-\alpha .
\end{aligned}
$$

Of course, a transformation $\omega_{\alpha}$ with $(\alpha, \alpha)=0$ cannot be lifted to an automorphism of the B.S.A. since even (respectively odd) roots being transformed by $\omega_{\alpha}$ into 
odd (respectively even) ones, the grading would not be respected. However such transformations can be simply used to deduce from one simple root system $B$ all the other, unequivalent under $W(\mathscr{G})$, bases. The method is as follows: construct from any $\alpha \in B$ such that $(\alpha, \alpha)=0$ the system $\omega_{\alpha}(B)$, and then repeat the same operation on the obtained systems until no new basis arises. Now it is an easy job (at least for not-too-high-rank superalgebras) to construct from a given Dynkin diagram all the other ones, once noticing that only the roots linked to the grey root with respect to which the root system is transformed will be affected. For example a white dot will become a grey or a black one depending on the number of links and the direction of the arrow between the white dot and the grey dot associated to the transformation. Examples are given in Table 1 where all the Dynkin diagrams for the unitary and orthosymplectic superalgebras of rank $\leqq 4$ are constructed. The general form of Dynkin diagrams of the B.S.A. is presented in Table 2. In this table the small black dot (in the unitary and orthosymplectic series) represents either a white dot $\bigcirc$ or a grey one $O$, and $\mathrm{K}$ is the parity of the number of grey dots.

\section{Regular Semi-Simple Subsuperalgebras of a B.S.A.}

The complete knowledge of the different Dynkin diagrams relative to a given superalgebra $\mathscr{G}$ will be used in this section for the construction of its regular semisimple sub(super)algebras. Considering the canonical decomposition

$$
\mathscr{G}=H \oplus \sum_{\alpha \in \Delta} \mathscr{G}_{\alpha},
$$

where $H$ is a Cartan subalgebra and $A=\Delta_{0} \cup \Delta_{1}$ its corresponding root system, a subsuperalgebra $\tilde{\mathscr{G}}$ is called regular, by analogy with the Lie algebra case, if it is such that:

$$
\tilde{\mathscr{G}}=\tilde{H} \oplus \sum_{\gamma^{\prime} \in \tilde{A}} \mathscr{G}_{x^{\prime}}
$$

with $\tilde{H} \subset H$ and $\tilde{\Delta} \subset \Delta$. The semi-simplicity of $\widetilde{\mathscr{G}}$ will be insured if to each $\alpha \in \tilde{\Delta}$ then $-\alpha \in \tilde{\Delta}$ and $\tilde{H}$ is the linear closure of $\tilde{A}$.

The method for finding the semi-simple regular sub(super)algebras of a B.S.A. is completely analogous to the usual one for Lie algebras by means of extended Dynkin diagrams [7]. (Note that a very clear presentation of the techniques and an explicit classification for regular subalgebras can be found in [8].) But now, one has to consider all the Dynkin diagrams associated to the inequivalent simple root systems. A first classification of the regular semi-simple sub(super)algebras of basic B.S.A. and the proof of the method has been recently given in [9]. Therefore we will limit ourselves to summarize hereafter the used techniques.

Given for a B.S.A. a simple root system and the associated Dynkin diagram. we draw the extended Dynkin diagram by adding to it a dot corresponding to the lowest root. Now, deleting arbitrarily one or more than one dot of this extended diagram, will yield one Dynkin diagram or a set of disjointed Dynking diagrams corresponding to a regular semi-simple sub(super)algebra of $\mathscr{G}$. Indeed, taking away one or more roots, one is left with a set of linearly independent roots which 
Table 1. Dynkin diagrams of unitary and orthosymplectic superalgebras of rank 1, 2, 3, 4

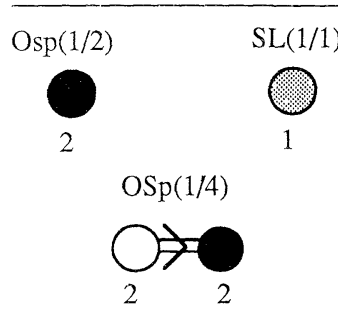

$\mathrm{OSp}(4 / 2)$

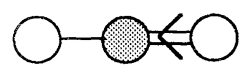

1

1
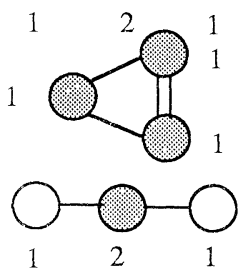

$\operatorname{OSp}(5 / 2)$
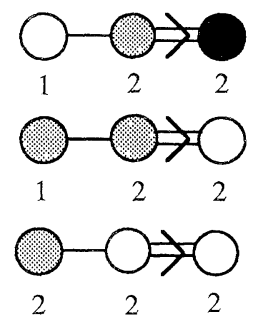

$\operatorname{OSp}(2 / 4)$

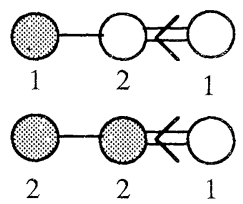

2
$\operatorname{OSp}(3 / 2)$

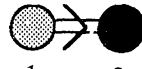

2

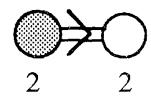

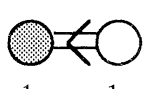

11

$\mathrm{OSp}(3 / 4)$

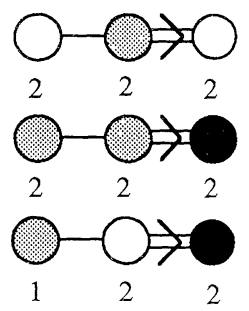

$\operatorname{OSp}(7 / 2)$
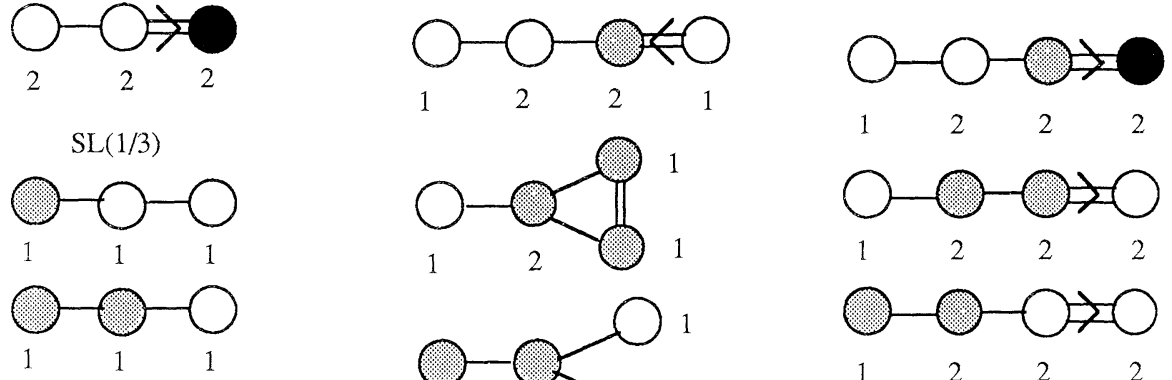

SL(2/2)
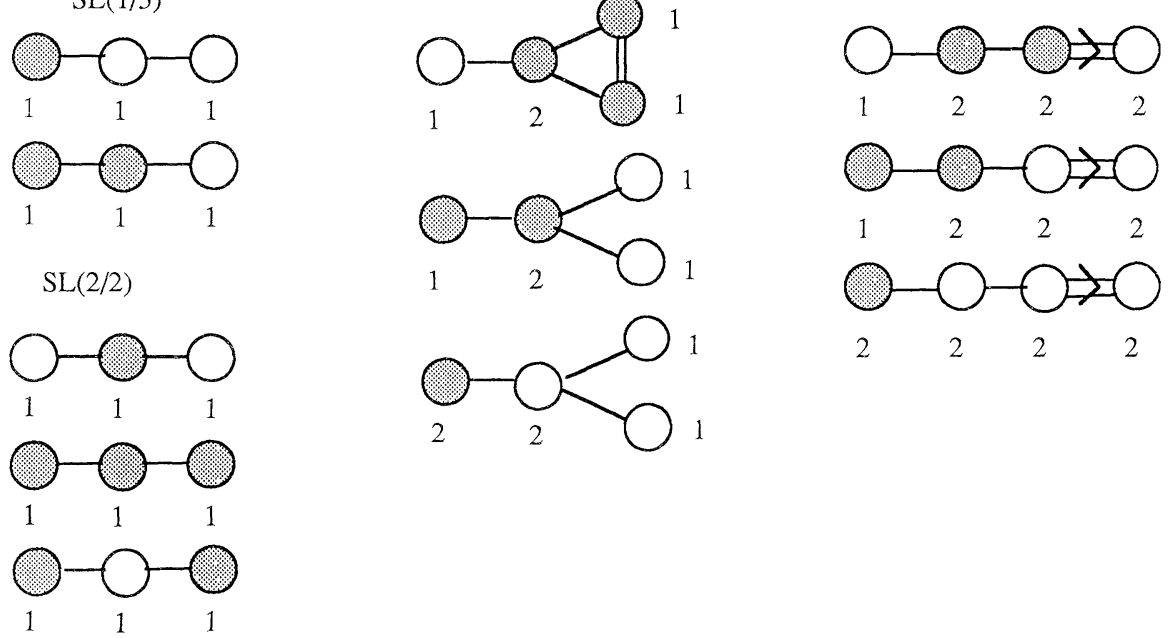
Table 1 (continued)
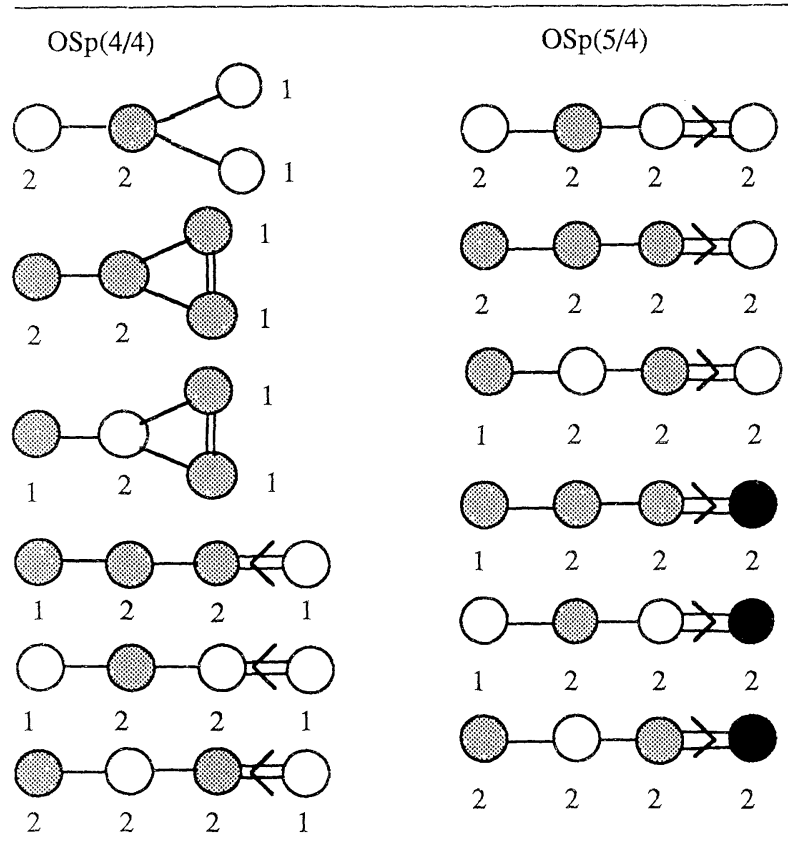

$\operatorname{OSp}(2 / 6)$
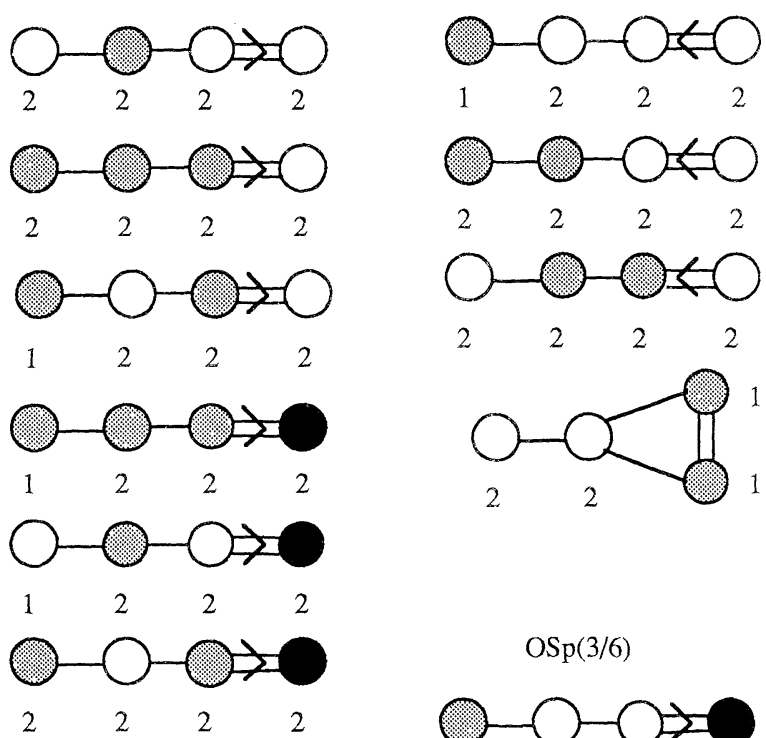

$\operatorname{OSp}(3 / 6)$

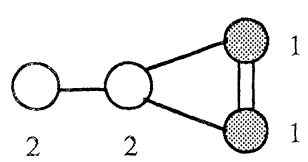

$\operatorname{SL}(2 / 3)$
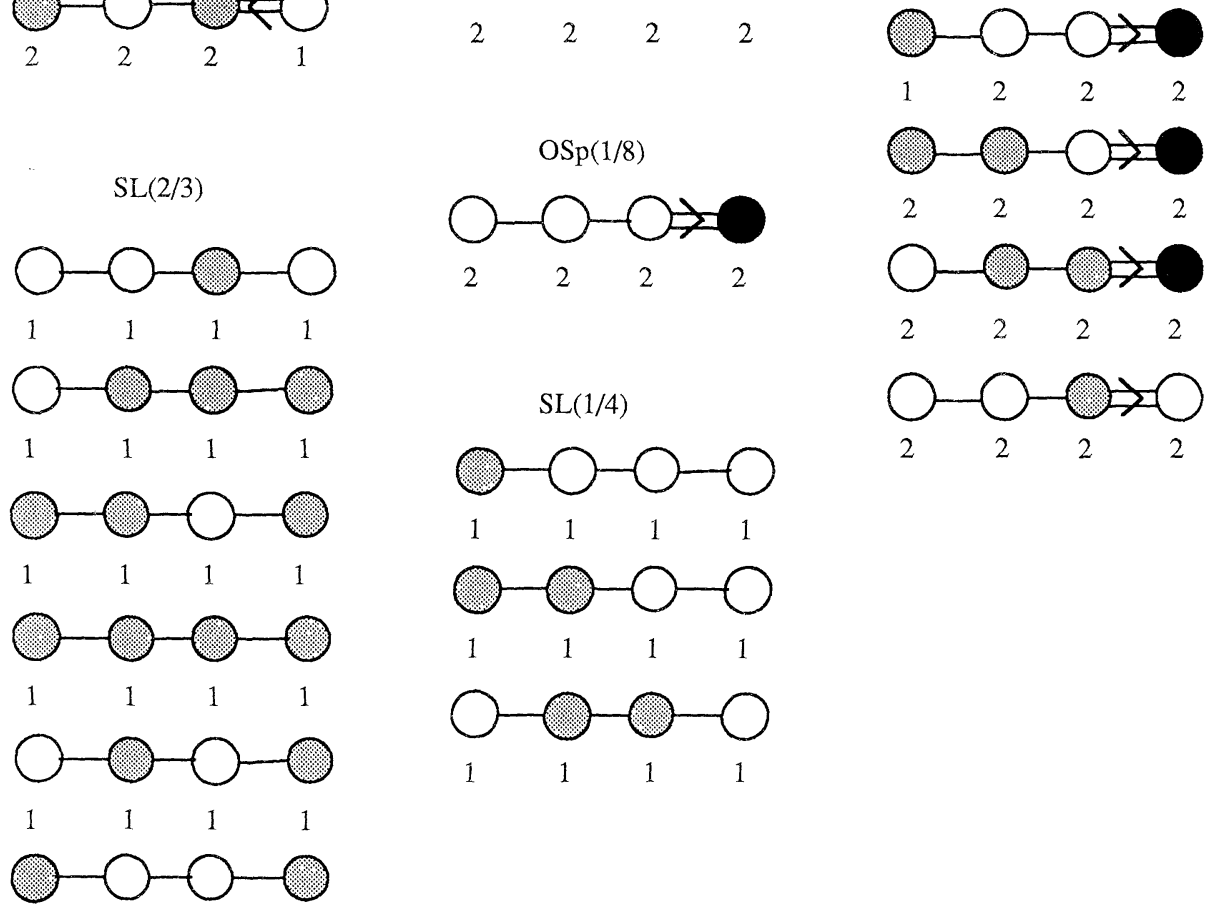

$\begin{array}{llll}1 & 1 & 1 & 1\end{array}$ 
Table 2. Dynkin diagrams of basic superalgebras

\section{$\operatorname{OSp}(2 m / 2 n)$}
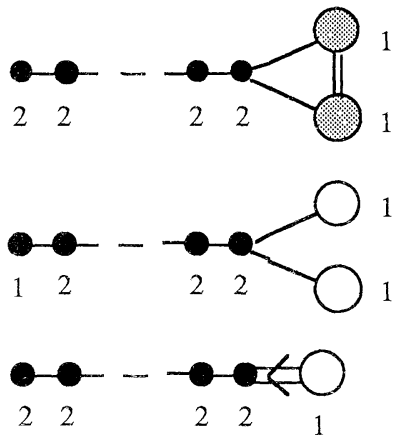

$$
K=0
$$

$\operatorname{OSp}(2 m+1 / 2 n)$
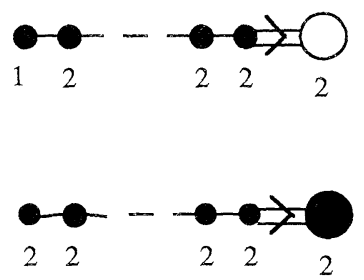

$$
\mathrm{K}=0
$$

\section{$\mathrm{SL}(\mathrm{m} / \mathrm{n})$}

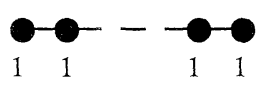

represents either

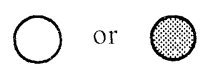

$\mathrm{K}$ is the parity of the number of

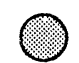

(see Sect. 3.2.3. for more details)

G(3)

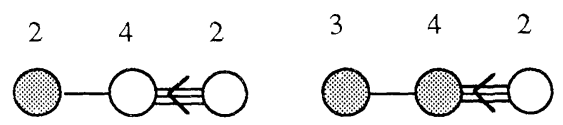

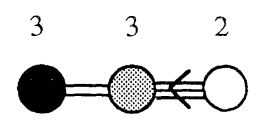


Table 2 (continued)

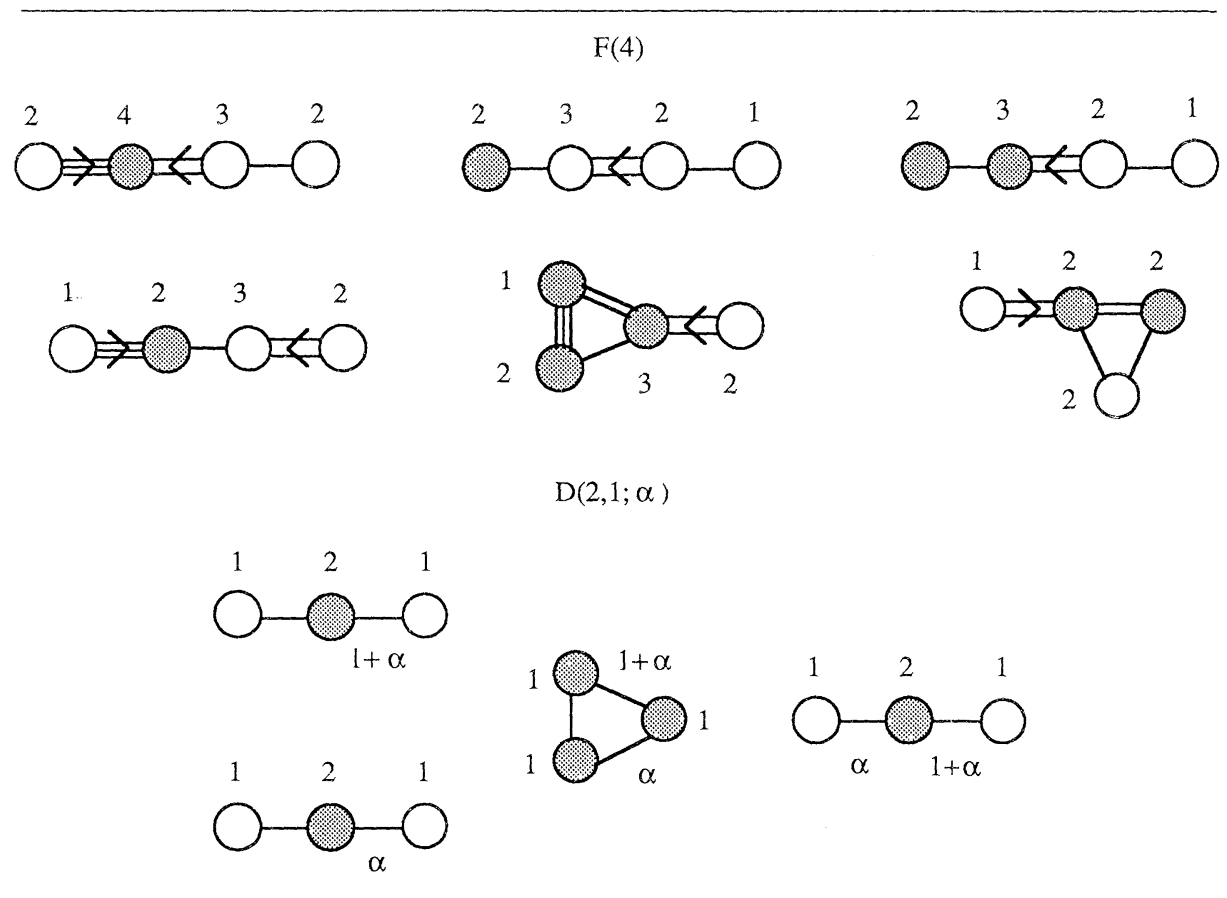

constitute the simple root system of a semi-simple regular sub(super)algebra of $\mathscr{G}$. Then repeating the same operation on the obtained Dynkin diagrams, i.e. adjunction of a dot associated to the lowest root of a simple part and cancellation of one arbitrary dot (or two dots in the unitary case) as many times as necessary, we will obtain in this way all the Dynkin diagrams associated with regular semisimple B.S.A. One can easily notice that in order to get the maximal regular semisimple B.S.A. of the same rank as $\mathscr{G}$, only the first step has to be achieved (which does not mean that all the so-obtained subsuperalgebras are maximal). The other possible maximal regular subsuperalgebras of $\mathscr{G}$ if they exist, will be obtained by deleting one dot in the (non-extended) Dynkin diagram of $\mathscr{G}$ and will be therefore of rank $r-1$. Examples are explicitly studied in Tables 3-6 while the list of maximal regular semi-simple subsuperalgebras for the B.S.A. is presented in Table 7.

\section{Outer Automorphisms of Superalgebras}

In the same way that outer automorphisms of a simple Lie algebra are related to symmetries of its corresponding Dynkin diagram, we can show that outer automorphisms of a B.S.A. can also be connected with some Dynkin diagram of $\mathscr{G}$. Let us recall [10] that in the case of a simple Lie algebra $\mathscr{A}$, one has the following isomorphisms:

$$
\frac{\operatorname{Aut}(\mathscr{A})}{\operatorname{Int}(\mathscr{A})} \simeq \frac{\operatorname{Aut}(\Delta)}{W(\Delta)}=F(\mathscr{A})
$$


Table 3. Maximal regular sub(super)algebras of $O S p(5 / 4)$
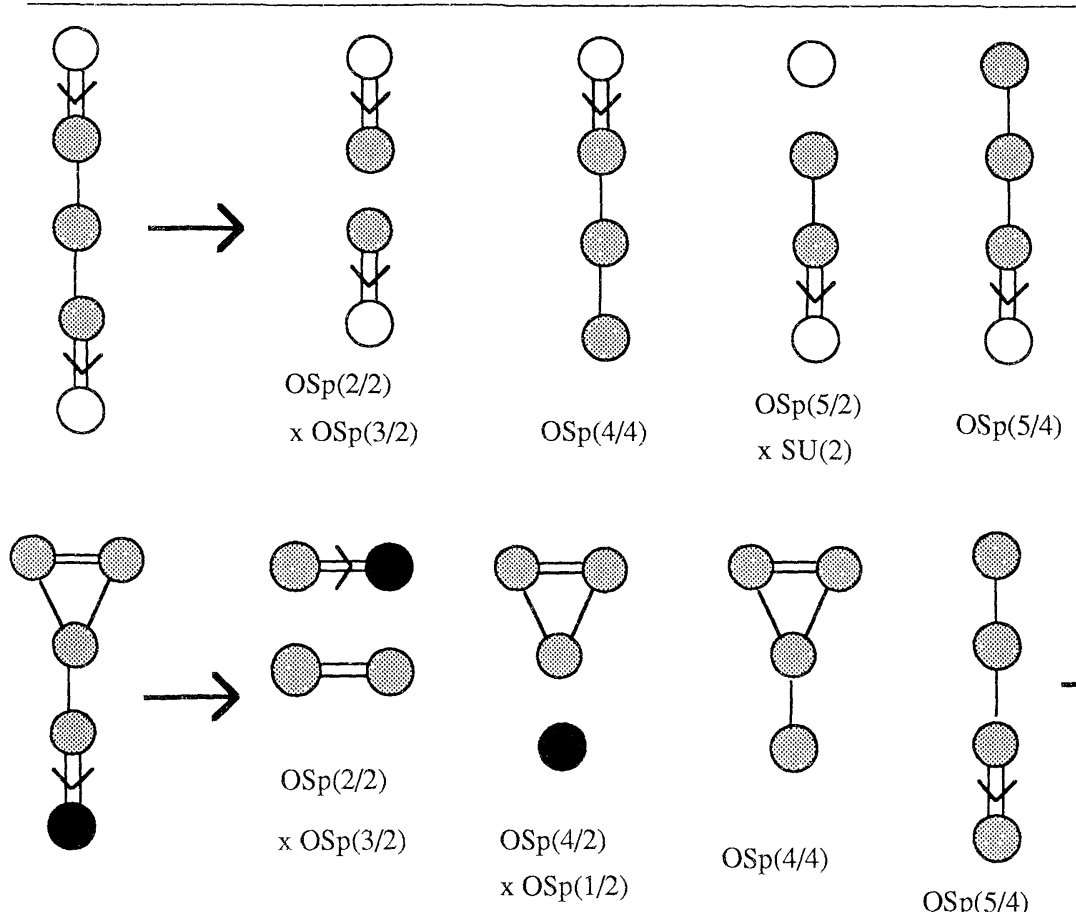

$\operatorname{OSp}(4 / 4)$

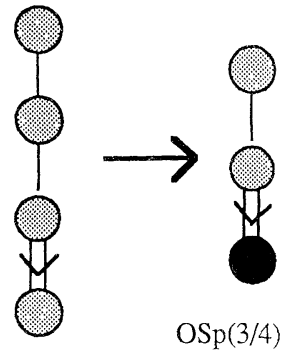

$\operatorname{OSp}(5 / 4)$
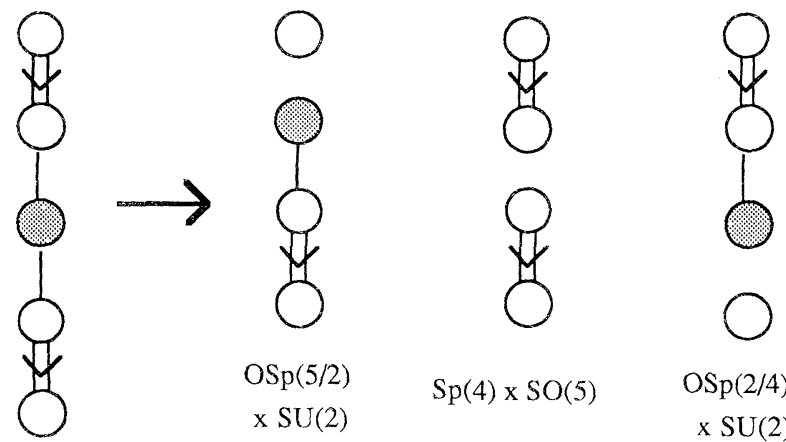

$\operatorname{OSp}(2 / 4)$

$x \mathrm{SU}(2)$
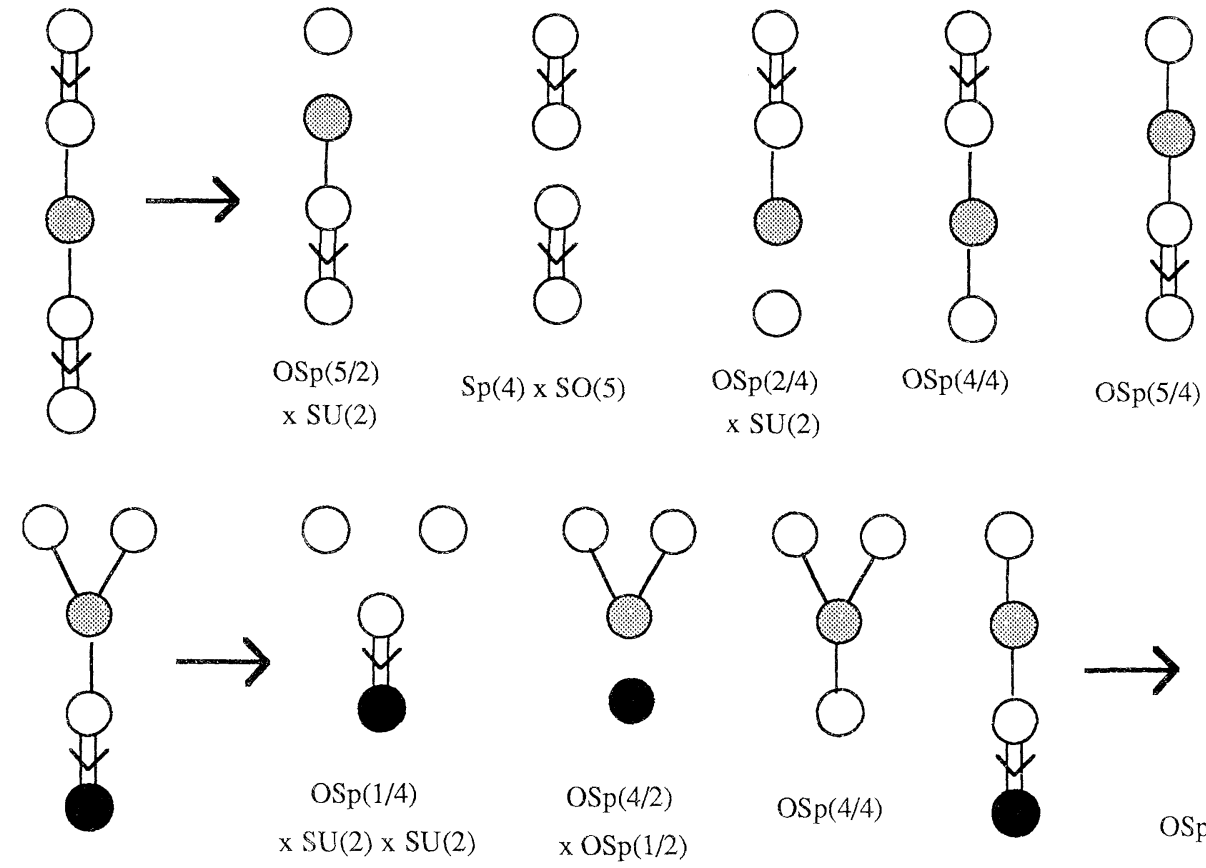

$\operatorname{OSp}(4 / 4)$

$\operatorname{OSp}(5 / 4)$
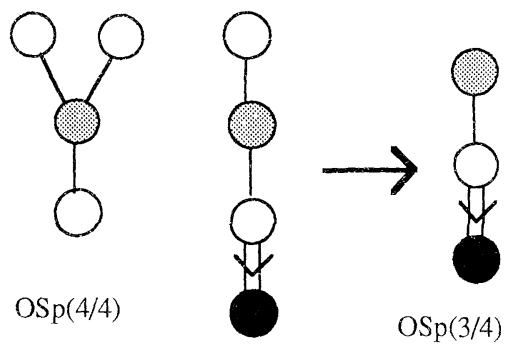

$\operatorname{OSp}(5 / 4)$ 
Table 3 (continued)

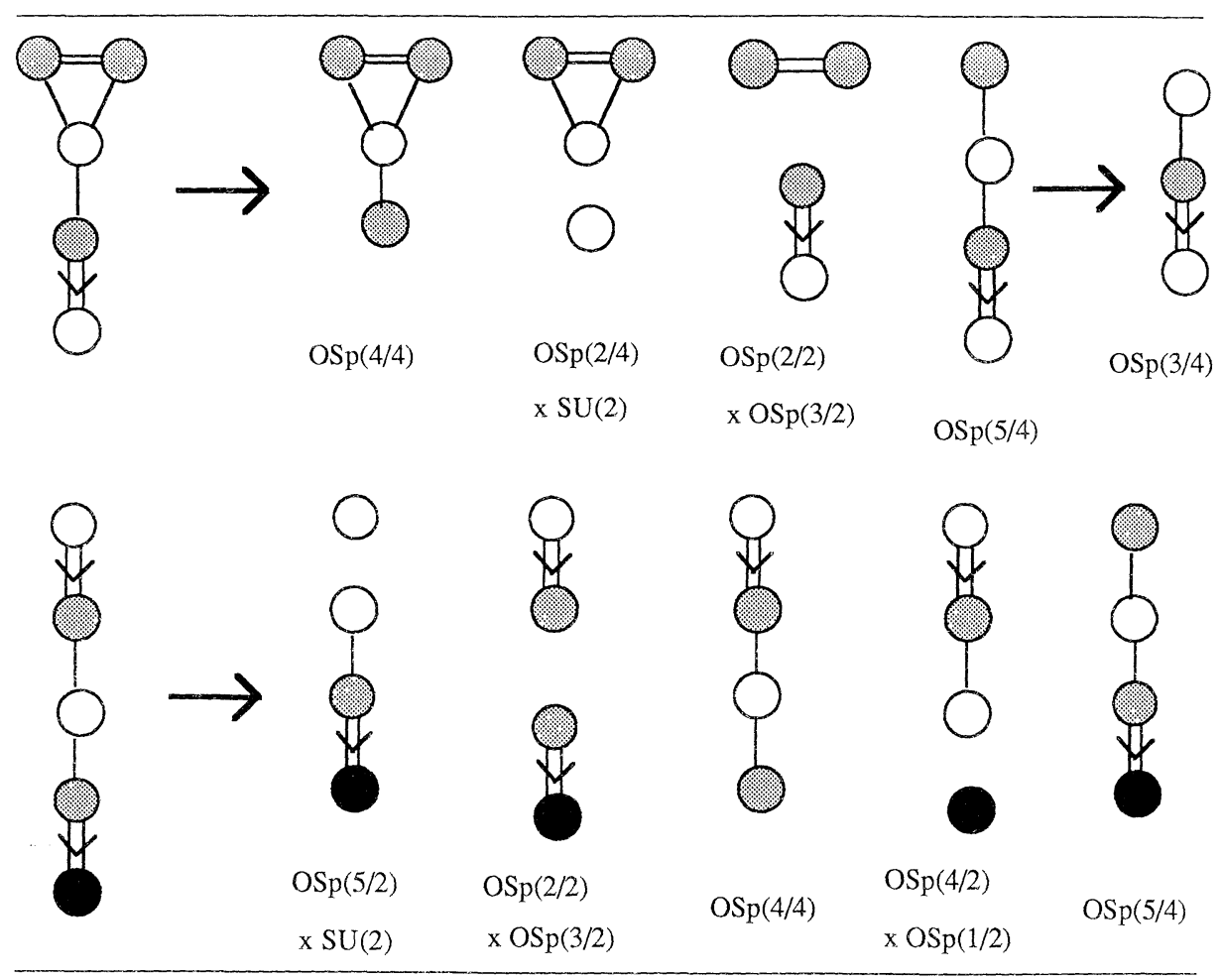

Table 4. Maximal regular sub(superalgebras) of $O S p(6 / 4)$

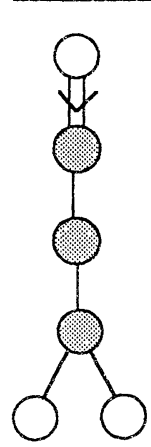

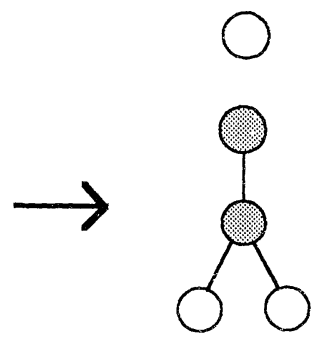

$\operatorname{OSp}(6 / 2)$

$\mathrm{x} \operatorname{SU}(2)$
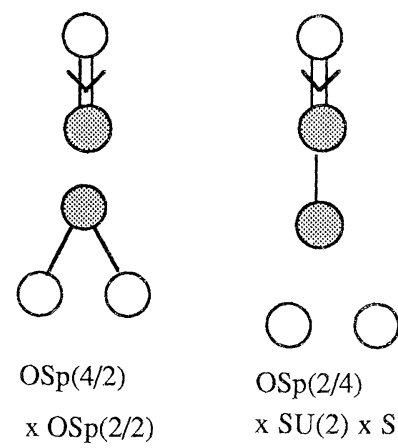

$\mathrm{OSp}(2 / 4)$

$x \mathrm{SU}(2) \times \mathrm{SU}(2)$

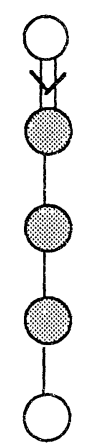

$\operatorname{OSp}(6 / 4)$ 
Table 4 (continued)
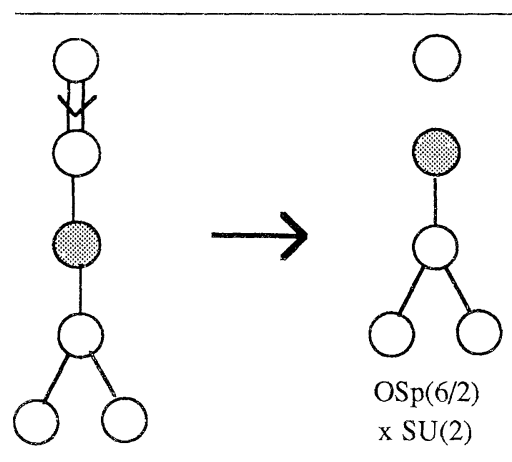

$\operatorname{OSp}(6 / 2)$

$\mathrm{x} \mathrm{SU}(2)$
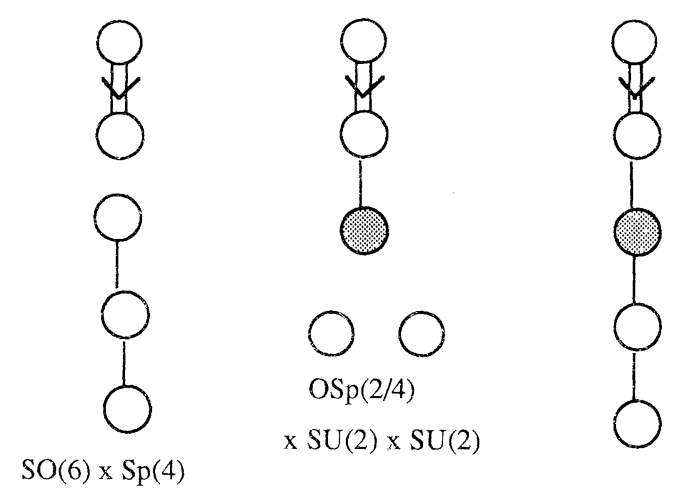

$\operatorname{OSp}(6 / 4)$

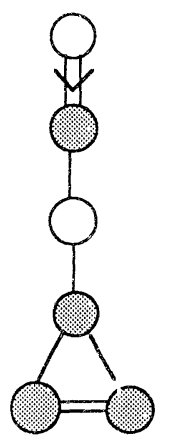

0

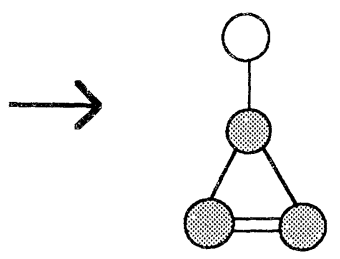

$\operatorname{OSp}(6 / 2)$

$\mathrm{x} \operatorname{SU}(2)$

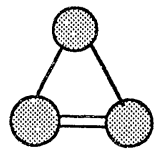

$\operatorname{OSp}(4 / 2)$

$\mathrm{x} \operatorname{OSp}(2 / 2)$
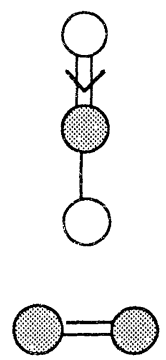

$\operatorname{OSp}(4 / 2)$

$x \operatorname{OSp}(2 / 2)$

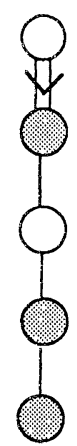

$\operatorname{OSp}(6 / 4)$

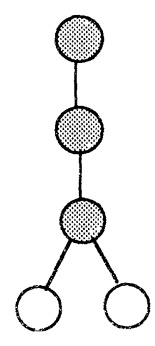

$\operatorname{OSp}(6 / 4)$

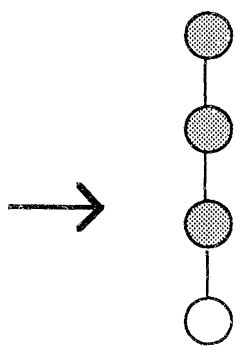

$\operatorname{SL}(3 / 2)$

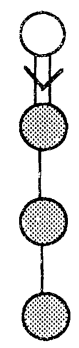

$\mathrm{OSp}(4 / 4)$ 
Table 4 (continued)
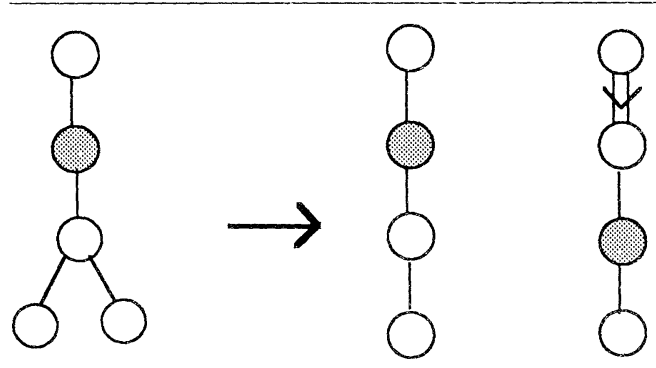

$\operatorname{SL}(3 / 2)$

$\operatorname{OSp}(6 / 4)$
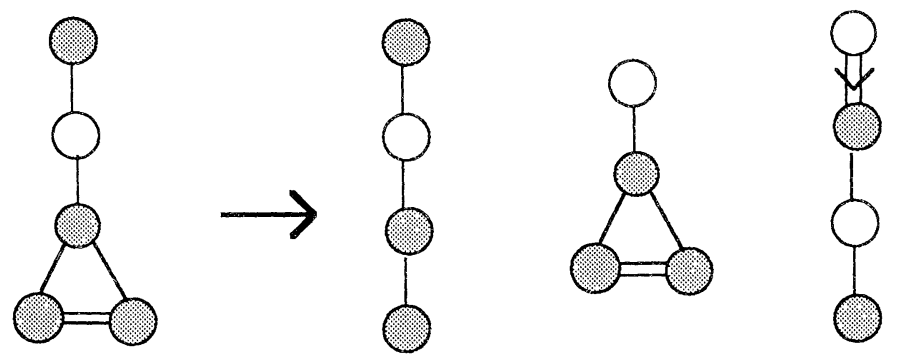

$\operatorname{OSp}(6 / 4)$

$\mathrm{SL}(3 / 2)$

$\operatorname{OSp}(4 / 4)$

$\operatorname{OSp}(4 / 4)$
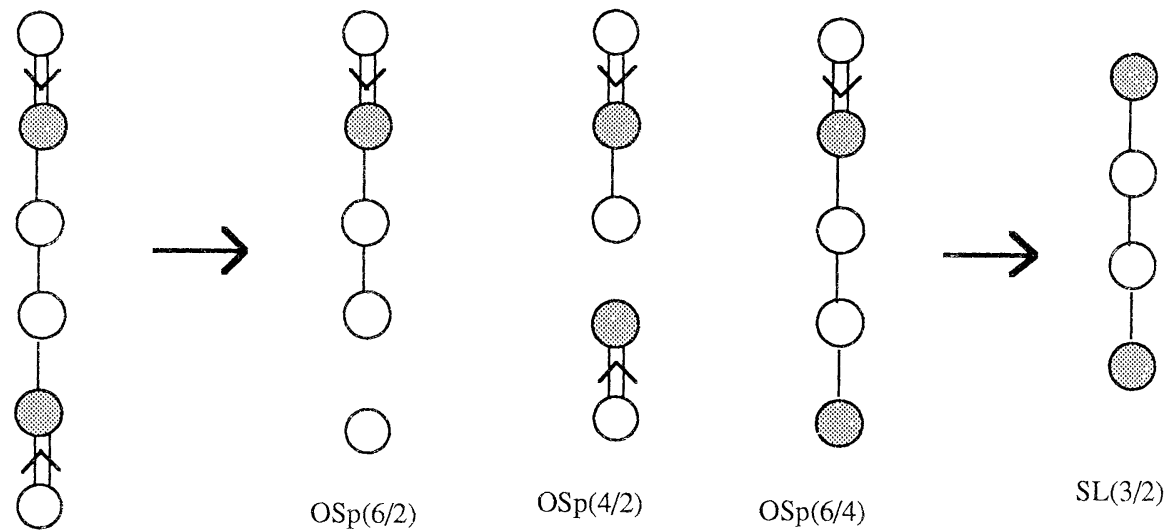

$\operatorname{OSp}(6 / 2)$

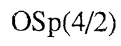

$\operatorname{OSp}(6 / 4)$

$\operatorname{SL}(3 / 2)$ 
Table 4 (continued)
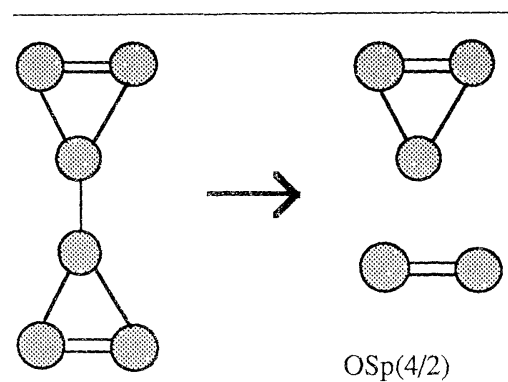

$\operatorname{OSp}(4 / 2)$

$x \operatorname{OSp}(2 / 2)$
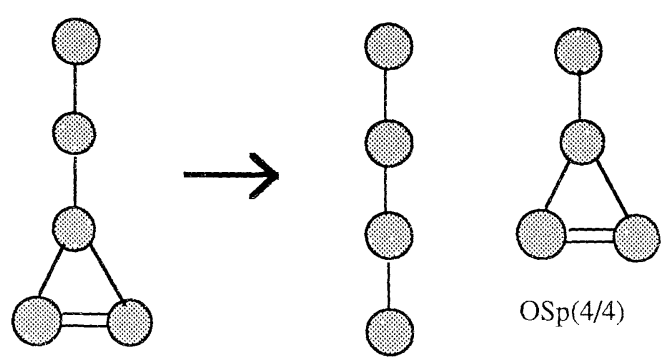

$\operatorname{OSp}(4 / 4)$

$\operatorname{OSp}(6 / 4)$

SL $(3 / 2)$
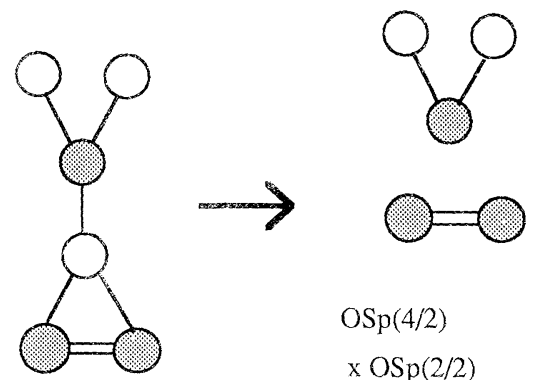

$x \operatorname{OSp}(2 / 2)$

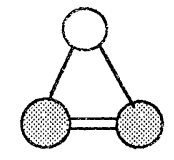

$\operatorname{OSp}(2 / 4)$

$x \operatorname{SU}(2) \times \operatorname{SU}(2)$

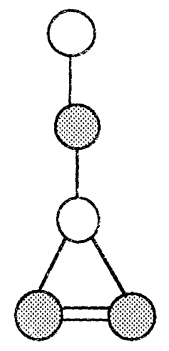

OSp(6/4)

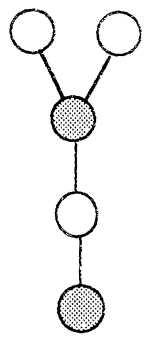

$\operatorname{OSp}(6 / 4)$
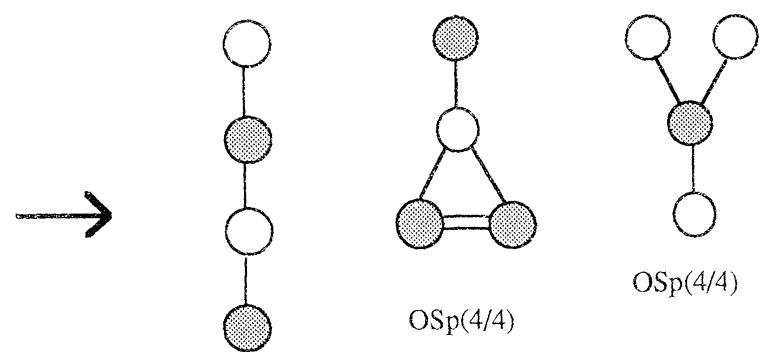

$\operatorname{OSp}(4 / 4)$ 
Table 5. Maximal regular sub(superalgebras) of $G(3)$

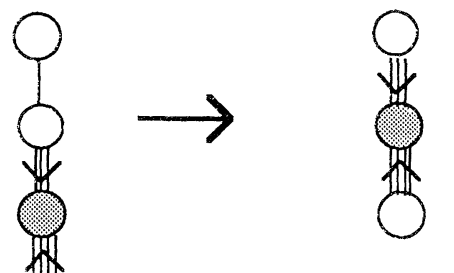

$\operatorname{OSp}(4 / 2 ; 3)$

III $\rightarrow$

莫

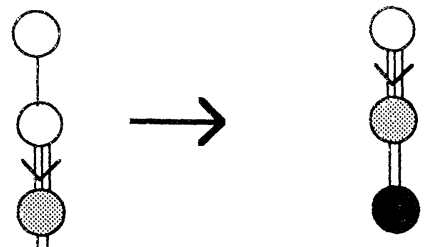

$\mathrm{G}(3)$

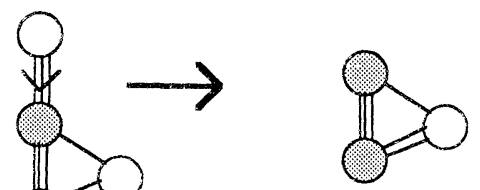

G(3)

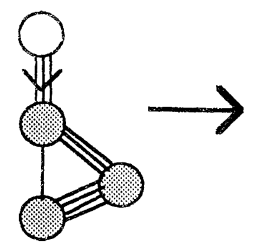

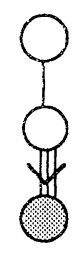

$\operatorname{SL}(1 / 3)$
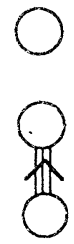

$\mathrm{SU}(2) \times \mathrm{G}_{2}$
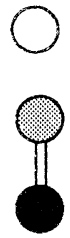

$\operatorname{OSp}(3 / 2)$

$\mathrm{x} \mathrm{SU}(2)$

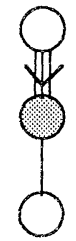

$\operatorname{OSp}(4 / 2 ; 3)$

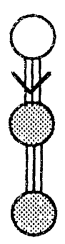

$\mathrm{G}(3)$

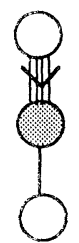

$\operatorname{OSp}(4 / 2 ; 3)$

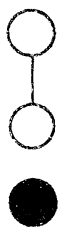

$\operatorname{OSp}(1 / 2)$

$\mathrm{x} \mathrm{SU}(3)$

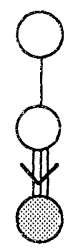

$\operatorname{SL}(1 / 3)$

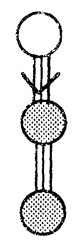

$\mathrm{G}(3)$

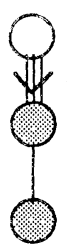

G(3) 
Table 6. Maximal regular sub(super)algegras of $F(4)$
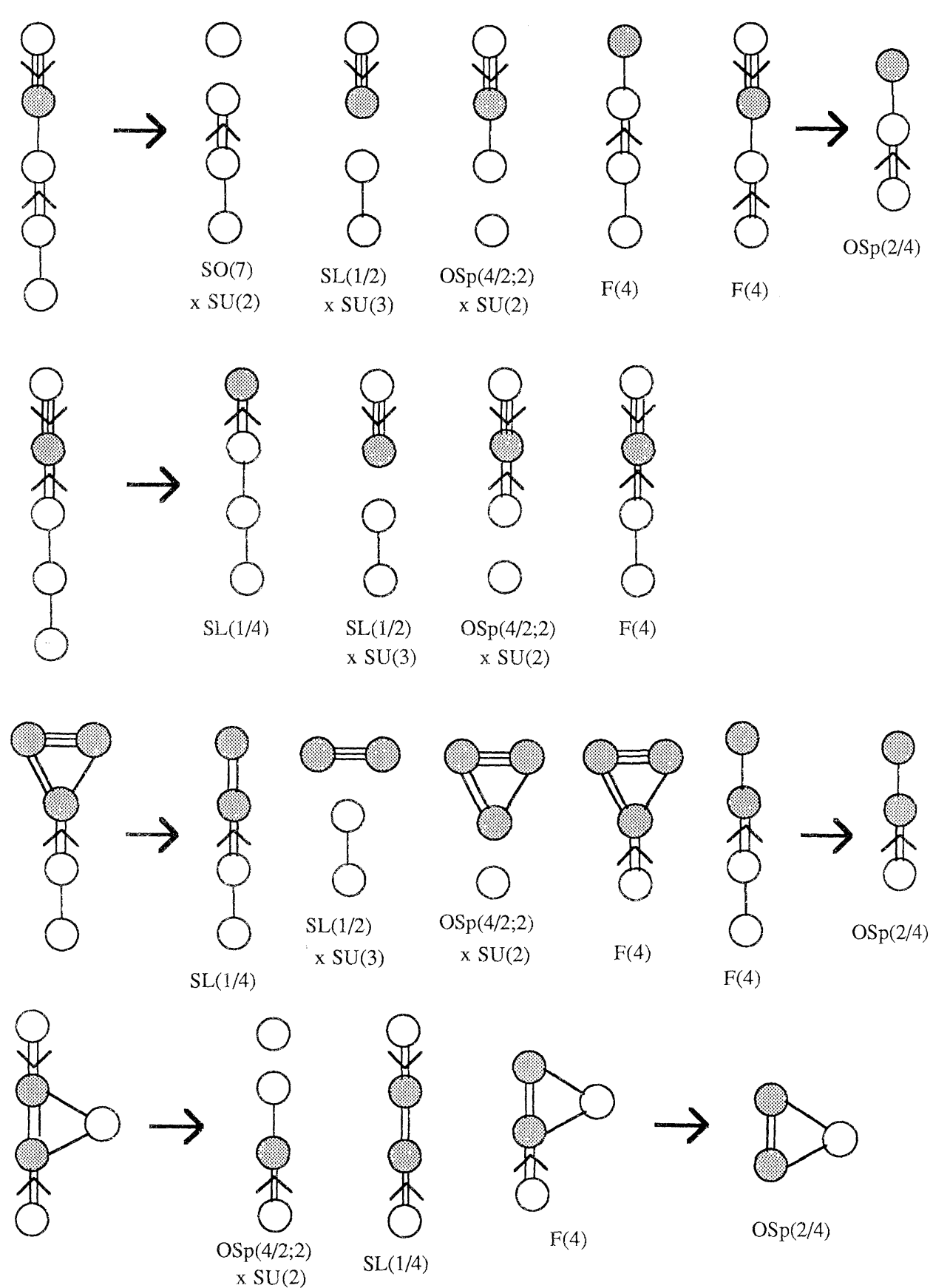

$\mathrm{F}(4)$

$\mathrm{F}(4)$ 
Table 7. Maximal regular subsuperalgebras of the B.S.A.

\begin{tabular}{|c|c|c|}
\hline$G$ & $\tilde{\mathscr{G}}$ & \\
\hline$A(m, n)$ & $\begin{array}{l}A(k, i) \oplus A(l, j) \\
A_{m} \oplus A_{n}\end{array}$ & $k+l=m, \quad i+j=n$ \\
\hline$B(m, n)$ & $\begin{array}{l}B(k, i) \oplus D(l, j) \\
B_{m} \oplus C_{n} \\
D(m, n)\end{array}$ & $k+l=m, \quad i+j=n$ \\
\hline$C(n+1)$ & $\begin{array}{l}C_{k} \oplus C(l+1) \\
C_{n}\end{array}$ & $k+l=n$ \\
\hline$D(m, n)$ & $\begin{array}{l}D(k, i) \oplus D(l, j) \\
D_{m} \oplus C_{n} \\
A(m, n)\end{array}$ & $k+l=m, \quad i+j=n$ \\
\hline$G(3)$ & $\begin{array}{l}A_{1} \oplus G_{2} \\
A_{1} \oplus B(1,1) \\
A_{2} \oplus B(0,1) \\
A(0,2) \\
D(2,1 ; 3) \\
G(3)\end{array}$ & \\
\hline$F(4)$ & $\begin{array}{l}A_{1} \oplus B_{3} \\
A_{2} \oplus A(0,1) \\
A_{1} \oplus D(2,1 ; 2) \\
A(0,3) \\
C(3) \\
F(4)\end{array}$ & \\
\hline$D(2,1 ; \alpha)$ & $\begin{array}{l}A_{1} \oplus A_{1} \oplus A_{1} \\
A(0,1) \\
D(2,1 ; \alpha)\end{array}$ & \\
\hline
\end{tabular}

where $\operatorname{Aut}(\mathscr{A})$ (respectively Int $(\mathscr{A})$ ) denotes the group of automorphisms (respectively inner automorphisms) of $\mathscr{A}$. Aut $(\Delta)$ is the group of automorphisms of the set of non-zero roots $\Delta$ of $\mathscr{A}$ with respect to a Cartan subalgebra $H$ and $W(\Delta)$ the Weyl group of $\Delta, F(\mathscr{A})$ is called the factor group and is therefore isomorphic to $\operatorname{Out}(\mathscr{A})$ if we define Out $(\mathscr{A})$ as the group of $\mathscr{A}$-automorphisms up to an element of $\operatorname{Int}(\mathscr{A})$, itself isomorphic to $\mathscr{A}$ since $\mathscr{A}$ is simple. $F(\mathscr{A})$ is isomorphic to the group of automorphisms of the Dynkin diagram and is simply the cyclic group of order two $Z_{2}$ for the algebras $A_{l}(l \geqq 2), D_{l}(l \geqq 4)$ and $E_{6}$, the cyclic group of order three $Z_{3}$ for the $D_{4}$ case while it reduces to the identity for the algebras $A_{1}, B_{l}(l \geqq 2), E_{7}, E_{8}, F_{4}, G_{2}$. Actually the Weyl group transforms a basis or simple root system of $\mathscr{A}$ into another basis in a transitive way, while $\operatorname{Aut}(\Delta(\mathscr{A})) / W(\Delta(\mathscr{A}))$ leaves a basis of $\mathscr{A}$ unchanged. In the same way an element of $W(\Delta)$ can be lifted to an inner automorphism of $\mathscr{A}$, a symmetry of the Dynkin diagram could be associated to an (outer)automorphism of $\mathscr{A}$. We illustrate the action of $F(\mathscr{A})$ on the Dynkin diagram of the $A_{n}$ algebras: 


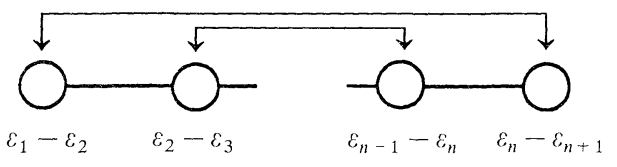

which corresponds to the transformation $\varepsilon_{i} \rightarrow-\varepsilon_{n+2-i},(i=1, \ldots, n+1)$.

The automorphisms of a basic superalgebra $\mathscr{G}$ have been worked out in $[4,11,12]$ and the results can be summarized as follows. Since any automorphism of a B.S.A. must respect the grading, it will act on the reductive bosonic part $\mathscr{G}_{0}$ of $\mathscr{G}$ as an automorphism of $\operatorname{Aut}\left(\mathscr{G}_{0}\right)$. Denoting Out $(\mathscr{G})=\operatorname{Aut}(\mathscr{G}) / \operatorname{Int}(\mathscr{G})$, where $\operatorname{Int}(\mathscr{G}) \simeq \mathscr{G}_{0}$, then Out $(\mathscr{G})$ is isomorphic to $Z_{2}$ for the superalgebras $A(m, n)$ (with $m \neq n$ and $m, n \neq 0), A(1,1), A(0,2 n+1)$ and $D(m, n)$ (with $m \neq 1$ ). It is isomorphic to $Z_{2} \times Z_{2}$ for $A(m, m)$ (with $\left.m \neq 0,1\right)$ and to $Z_{4}$ for $A(0,2 n)$. Out $(\mathscr{G})$ reduces to the identity for $B(m, n), C(n+1), F(4)$ and $G(3)$. Out $(D(2,1 ; \alpha))$ (with $\alpha \neq 0,-1)$ is trivial in general, except for the values $\alpha=1,-1 / 2,-2$, where it is isomorphic to $Z_{2}$ and $\alpha=e^{2 i \pi / 3}, e^{4 i \pi / 3}$, where it becomes isomorphic to the three element group $Z_{3}$. Out $(\mathscr{G})$ can be reconstructed in general by looking at the symmetries of the Dynkin diagrams of $\mathscr{G}$, except in some special cases.

Consider first the $S l(m \mid n)$ case. For all values of $m$ and $n$ (with $m \neq n$ and $m, n$ not simultaneously odd), it is always possible to construct a $Z_{2}$ symmetric Dynkin diagram as follows:

- $\operatorname{Sl}(2 m+1 \mid 2 n)$ with $m<n$

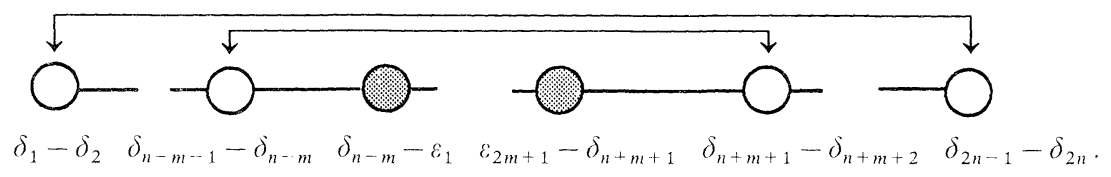

- $\operatorname{Sl}(2 m+1 \mid 2 n)$ with $m>n$

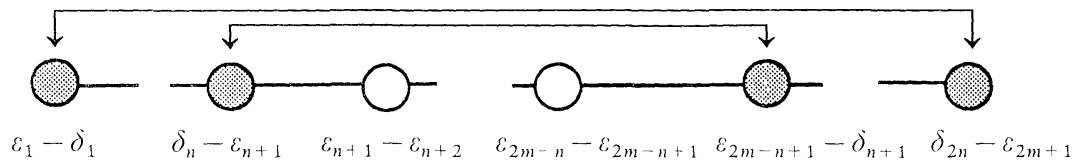

both cases corresponding to the same transformation

$$
\varepsilon_{i} \rightarrow-\varepsilon_{2 m+2-i}(i=1, \ldots, 2 m+1) \quad \text { and } \quad \delta_{j} \rightarrow-\delta_{2 n+1-j}(j=1, \ldots, 2 n) .
$$

- $\operatorname{Sl}(2 m+1 \mid 2 m)$

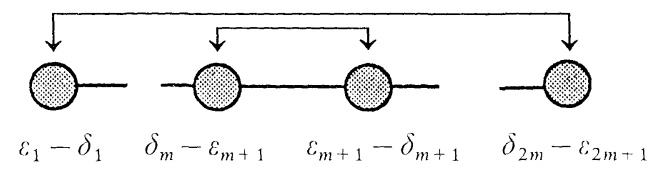

the transformation is here

$$
\varepsilon_{\imath} \rightarrow-\varepsilon_{2 m+2-\imath}(i=1, \ldots, 2 m+1) \quad \text { and } \quad \delta_{j} \rightarrow-\delta_{2 m+1-j}(j=1, \ldots, 2 m) .
$$


- $\quad \operatorname{Sl}(2 m \mid 2 n)$ with $m \neq n$

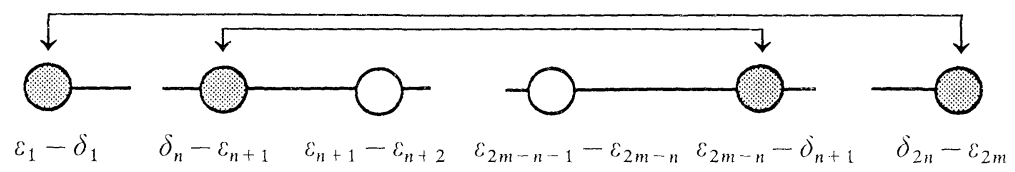

in which the transformation is

$$
\varepsilon_{i} \rightarrow-\varepsilon_{2 m+1-i}(i=1, \ldots, 2 m) \quad \text { and } \quad \delta_{j} \rightarrow-\delta_{2 n+1-j}(j=1, \ldots, 2 n)
$$

Then for the symplectic case, one has:

- $O S p(2 m \mid 2 n)$ with $m \neq 1$

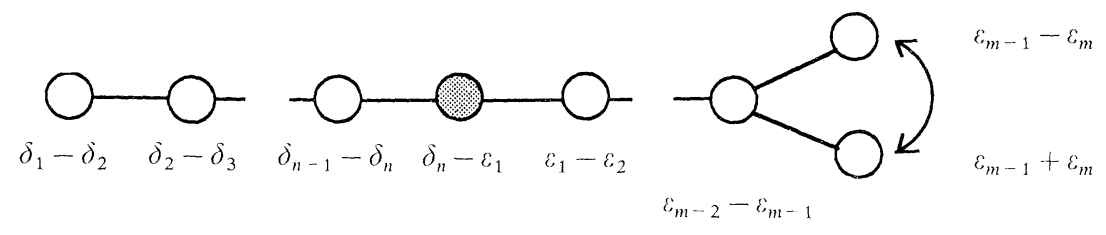

the transformation being $\varepsilon_{m} \rightarrow-\varepsilon_{m}$.

- $\operatorname{OSp}(2 \mid 2 n)$

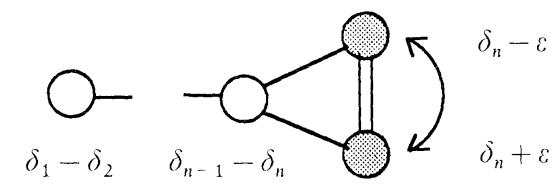

the transformation being here $\varepsilon \rightarrow-\varepsilon$.

At this point, one can notice that each symmetry $\tau$ described above induces a direct construction of the subsuperalgebra $\mathscr{G}^{\prime}$ invariant under the $\mathscr{G}$ outer automorphisms associated to $\tau$. Indeed if the simple root $\alpha$ is transformed into $\tau(\alpha)$, then $\frac{1}{2}(\alpha+\tau(\alpha))$ is $\tau$-invariant since $\tau^{2}=1$, and appears as a simple root of $\mathscr{G}^{\prime}$ associated to the generator $E_{\alpha}+E_{\tau(\alpha)}$, if $E_{\alpha}$ (respectively $E_{\tau(\alpha)}$ ) corresponds to the root $\alpha$ (respectively $\tau(\alpha)$ ). A Dynkin diagram of $\mathscr{G}^{\prime}$ will therefore be obtained by folding the $Z_{2}$ symmetric Dynkin diagram of $\mathscr{G}$, that is by transforming each couple $(\alpha, \tau(\alpha))$ into the $\operatorname{root} \frac{1}{2}(\alpha+\tau(\alpha))$ of $\mathscr{G}^{\prime}$. Let us add that this folding method has already been used in [13] in order to obtain the non-simply laced simple Lie algebras from the simply laced ones. One obtains here the following invariant subsuperalgebras 

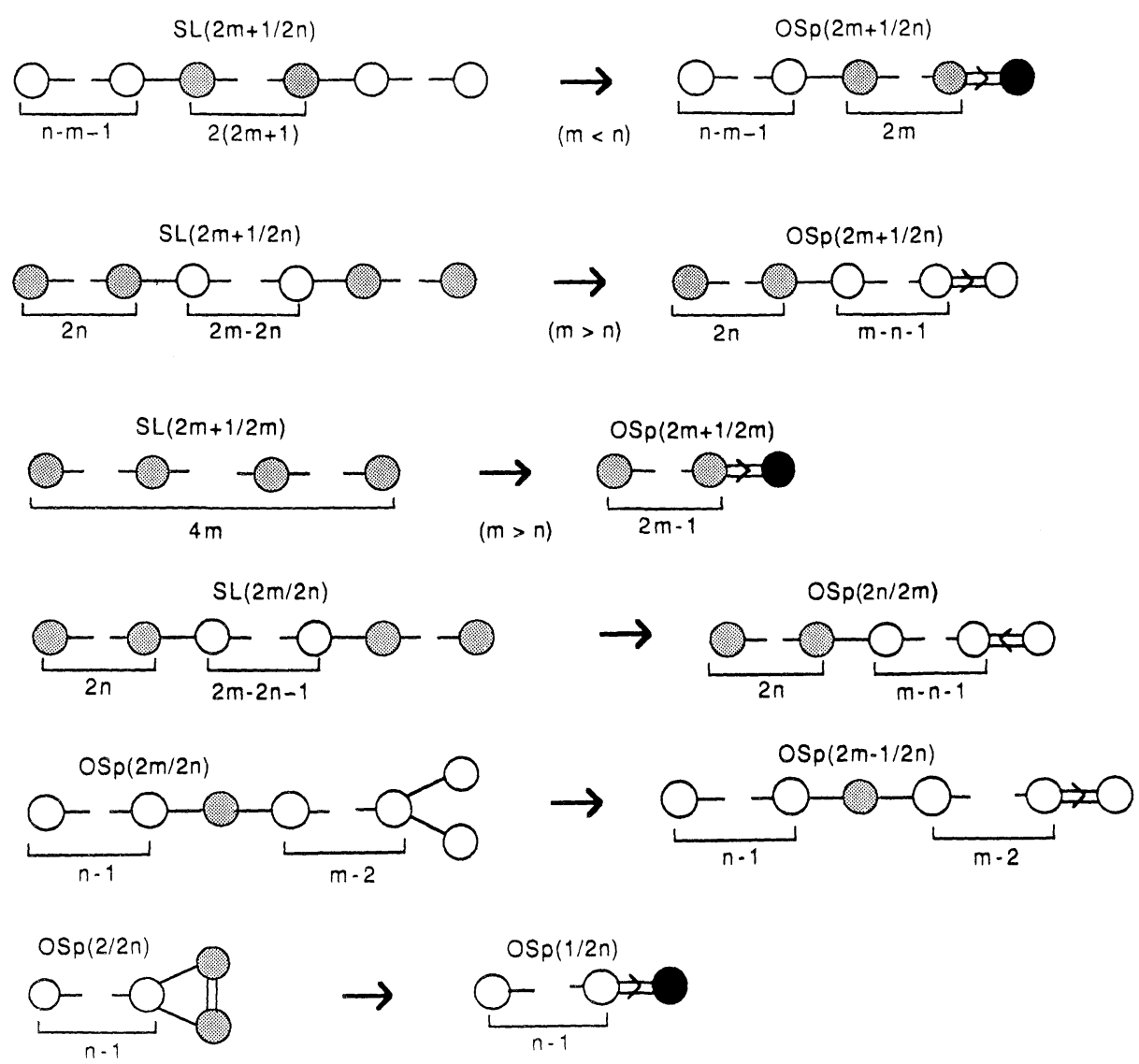

The previous method cannot be used in the case of $\operatorname{Sl}(2 m+1 \mid 2 n+1)(m \neq n)$ since there does not exist any symmetric Dynkin diagram associated to this type of superalgebra. However one notes a global symmetry of the set of Dynkin diagrams. For example, in the case of $S l(1 \mid 3)$, one has the following tableau:

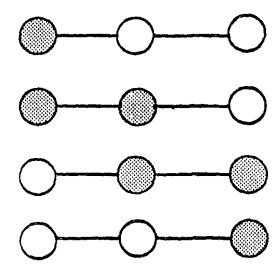

In the case of $\operatorname{Sl}(2 m+1 \mid 2 m+1)$, besides the global symmetry of the set of Dynkin diagrams, there exist a $Z_{2}$ symmetric diagram

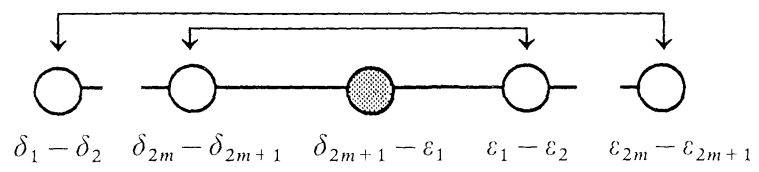


the transformation being $\varepsilon_{i} \rightarrow-\delta_{2 m+2-i}(i=1, \ldots, 2 m+1)$, associated to the permutation of the two $\operatorname{Sl}(2 m+1)$ subalgebras. However, the folding does not give any information: actually the invariant subsuperalgebra under the above symmetry is a strange simple superalgebra to which a Dynkin diagram cannot be associated.

In the case of $S l(2 m \mid 2 m)$, one finds a $Z_{2}$ symmetric Dynkin diagram

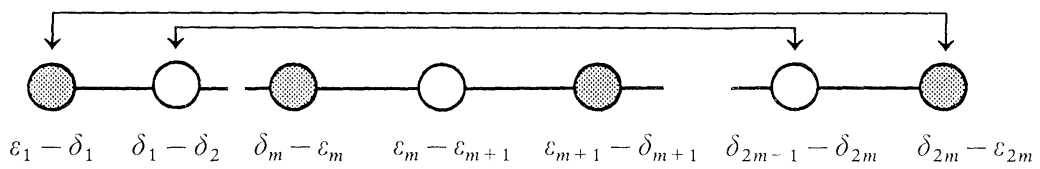

with the transformation $\varepsilon_{i} \rightarrow-\varepsilon_{2 m+1-i}$ and $\delta_{i} \rightarrow-\delta_{2 m+1-i}(i=1, \ldots, 2 m)$. By folding, this diagram leads to the invariant subsuperalgebra $O S p(2 m \mid 2 m)$ as in the general case of $\operatorname{Sl}(2 m \mid 2 n)$ :

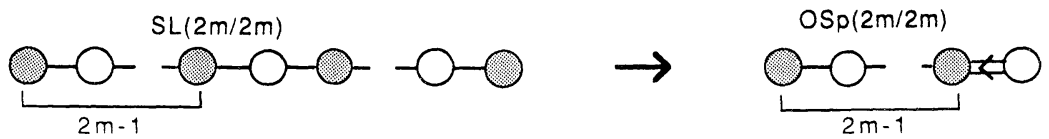

One notes also another $Z_{2}$ symmetric Dynkin diagram

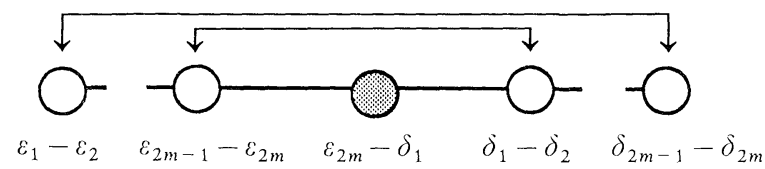

the transformation being $\varepsilon_{i} \rightarrow-\delta_{2 m+1-i}(i=1, \ldots, 2 m)$, associated to the interchange of the two $\operatorname{Sl}(2 m)$ subalgebras: as in the case of $S l(2 m+1 \mid 2 m+1)$ just discussed, the invariant subsuperalgebra is a strange one which cannot be obtained by folding.

The $D(2,1 ; \alpha)$ needs a little more attention. Using the corresponding Dynkin diagrams of Table 2 , one can observe a $Z_{2}$ symmetry on the linear diagrams for the values $\alpha=-2$ (corresponding to $|1+\alpha|=1$ ), $\alpha=1$ (corresponding to $|\alpha|=1$ ) and $\alpha=-1 / 2$ (corresponding to $|1+\alpha|=|\alpha|$ ). The triangular diagram has a $Z_{3}$ symmetry, generated by the circular permutations of 3 elements, for the values $\alpha=e^{2 i \pi / 3}, \alpha=e^{4 i \pi / 3}$. It might be interesting to consider in more detail this "triality" property.

\section{Untwisted and Twisted Affine Superalgebras}

\subsection{Untwisted Affine B.S.A.}

Properties on the structure of affine Lie superalgebras [14, 15] can also be deduced by extending the classification of Dynkin diagrams to the affine case. This will in particular allow us to construct in a diagrammatic way twisted affine superalgebras from untwisted ones. 
The definition of affine untwisted B.S.A. $\mathscr{G}^{(1)}$ follows that of affine algebras, i.e. $\mathscr{G}^{(1)}$ is the loop algebra constructed from $\mathscr{G}$ as

$$
\mathscr{G}^{(1)}=C\left(t, t^{-1}\right) \otimes \mathscr{G} \oplus C c,
$$

where $C\left(t, t^{-1}\right)$ stands for the algebra of Laurent polynomials in the complex variable $t$ and $c$ is the central extension term. The generators of $\mathscr{G}^{(1)}$ satisfy the commutation relations:

$$
\left[t^{m} \otimes a, t^{n} \otimes b\right]=t^{m+n} \otimes[a, b]+m(a, b) \delta_{m+n, 0} c,
$$

using relations of Sect. 2 .

A simple root system of an affine B.S.A. $\mathscr{G}^{(1)}$ is obtained from a simple root system $B$ of $\mathscr{G}$ by adding to it the affine root which project on $B$ as the corresponding lowest root. The simple root systems of $\mathscr{G}^{(1)}$ are therefore associated to the extended Dynkin diagrams used to determine the regular subsuperalgebras studied in Sect. 3. The Dynkin diagrams for the affine B.S.A. of rank less than 5 of the unitary and orthosymplectic series are constructed in Table 8, whereas their general form for all the B.S.A is given in Table 9.

\subsection{Twisted Affine B.S.A.}

Twisted Lie superalgebras $\mathscr{G}^{(m)}(m \neq 1)$ are associated with outer automorphisms $\hat{\tau}$ of $\mathscr{G}, m$ being the smallest positive integer such that $\hat{\tau}^{m}=1 . \mathscr{G}^{(m)}$ is a $\mathscr{G}^{(1)}$ subsuperalgebra, the elements of which are $\mathscr{G}$-valued functions

$$
t^{n} \otimes a \equiv|t|^{n} e^{i n \theta} \otimes a \equiv f(\theta) \otimes a
$$

submitted to the condition:

$$
f(\theta+2 \pi) \otimes a=f(\theta) \otimes \hat{\tau}(a) .
$$

Decomposing $\mathscr{G}$ with respect to the $\hat{\tau}$-eigenvalues $e^{2 i \pi k / m}(k=0, \ldots, m-1)$ one has

with

$$
\mathscr{G}=\bigoplus_{k=0}^{m-1} \hat{\mathscr{G}}_{k}
$$

$$
\hat{\mathscr{G}}_{k}=\left\{a \in \mathscr{G} \mid \hat{\tau}(a)=e^{2 i \pi k / m} a\right\} .
$$

satisfying the $Z / m Z$ gradation:

$$
\left[\hat{\mathscr{G}}_{h}, \hat{\mathscr{G}}_{l}\right] \subset \hat{\mathscr{G}}_{k+l}(\bmod m),
$$

while $\hat{\mathscr{G}}_{0}$ is the $\hat{\tau}$-invariant subsuperalgebra of $\mathscr{G}$. The decomposition for $\mathscr{G}^{(m)}$ follows:

$$
\mathscr{G}^{(m)}=\bigoplus_{\substack{k=0 \\ n \in Z}}^{m-1} t^{n+k / m} \otimes \hat{\mathscr{G}}_{k} .
$$

The Dynkin diagrams for the twisted affine B.S.A. can be constructed directly by the following method. For a twisted B.S.A. $\mathscr{G}^{(2)}$ related to an outer automorphism of order 2 , one can decompose $\mathscr{G}^{(2)}$ as a $\hat{\tau}$-invariant sub- 
Table 8. Dynkin diagrams of affine unitary and orthosymplectic superalgebras of rank 1,2,3,4,5
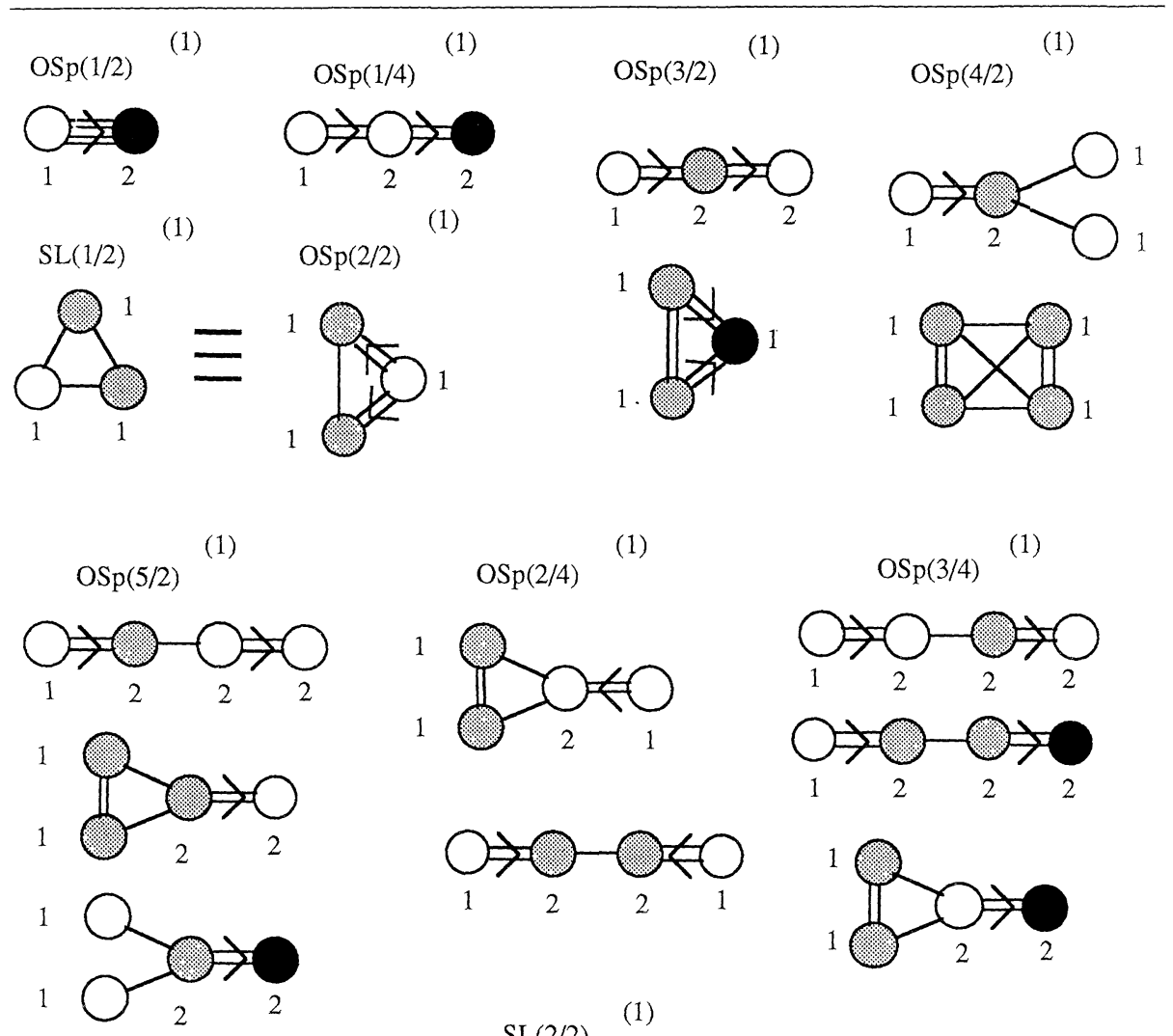

$\operatorname{SL}(2 / 2)$

(1)

$\operatorname{OSp}(1 / 6)$
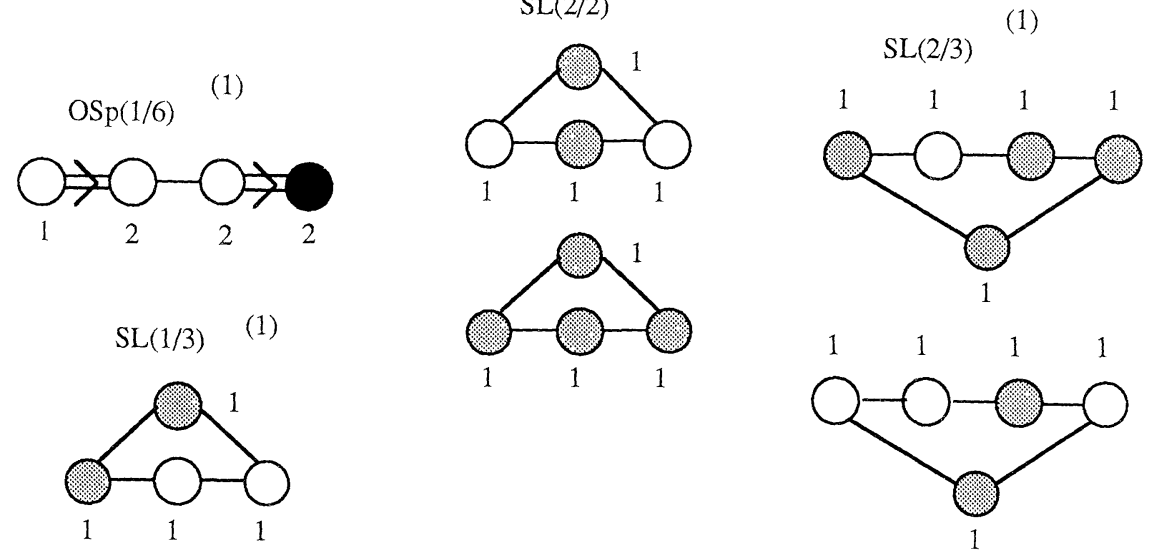
Table 8 (continued)

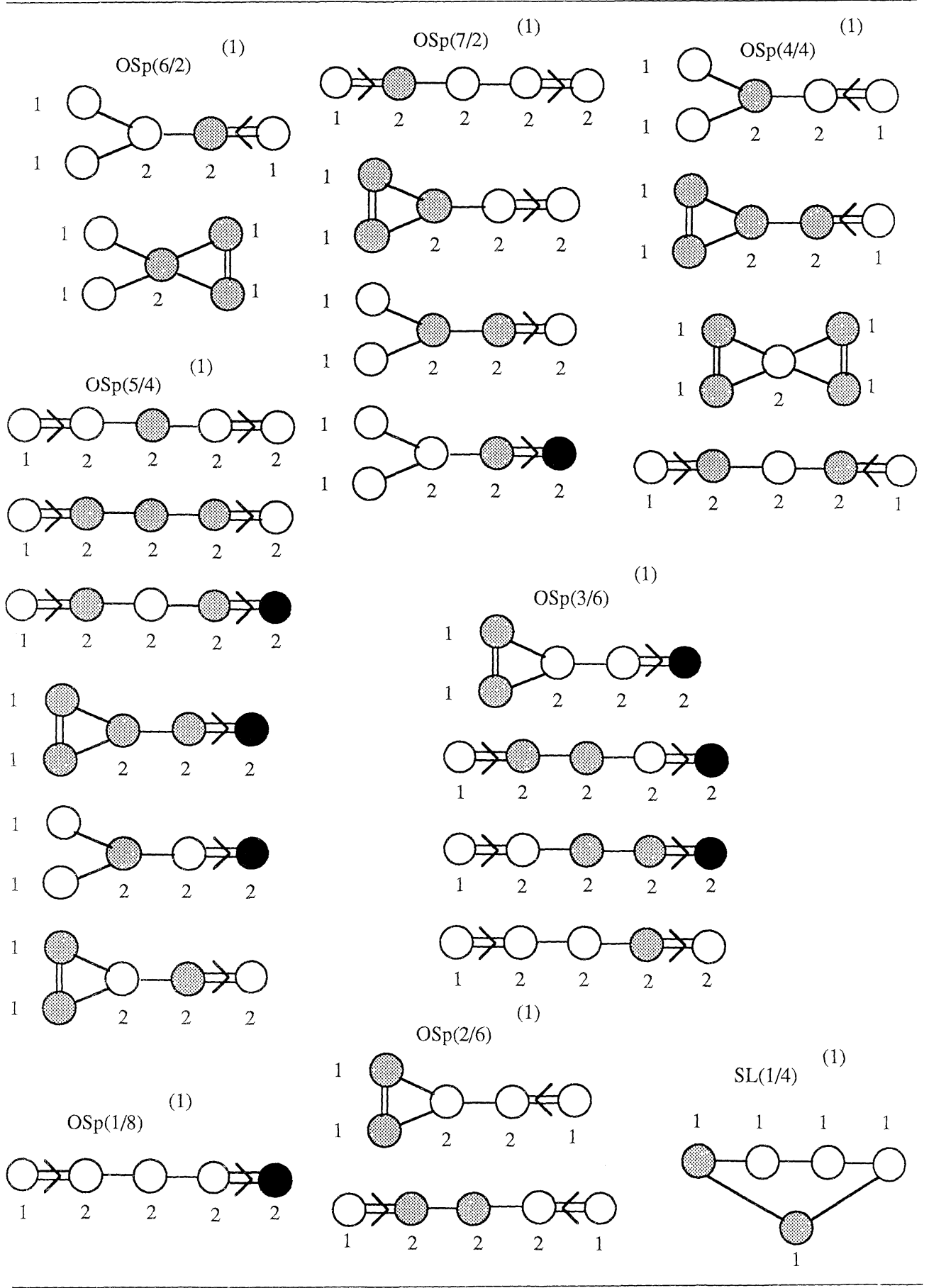


Table 9. Dynkin diagrams of affine B.S.A.

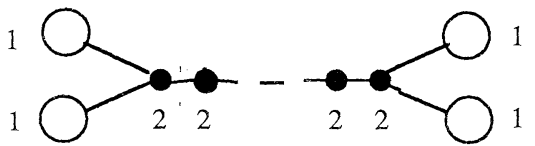

$\operatorname{OSp}(2 m / 2 n)$

1
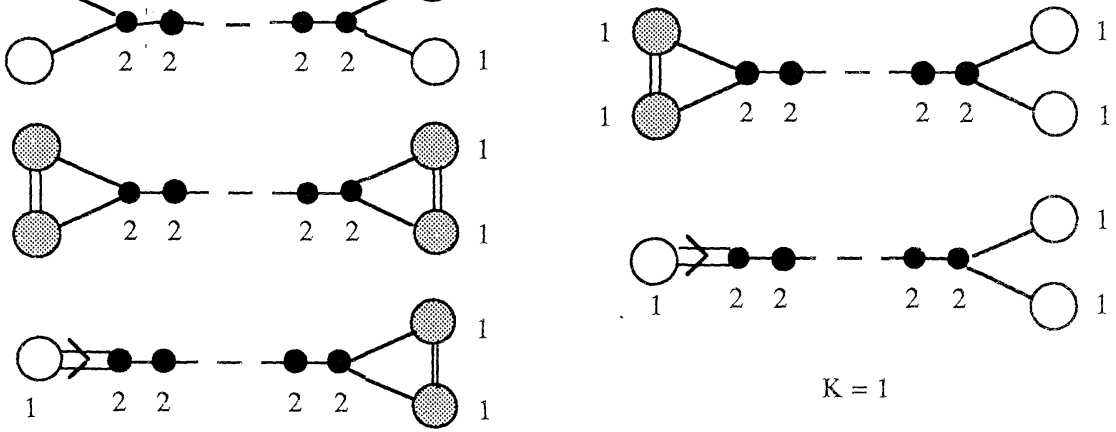

$\mathrm{K}=1$

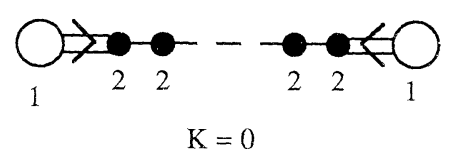

$\operatorname{OSp}(2 m+1 / 2 n)$

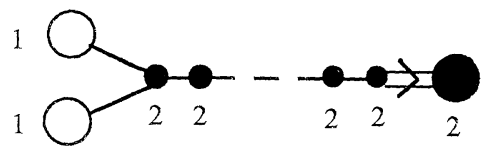

(1)
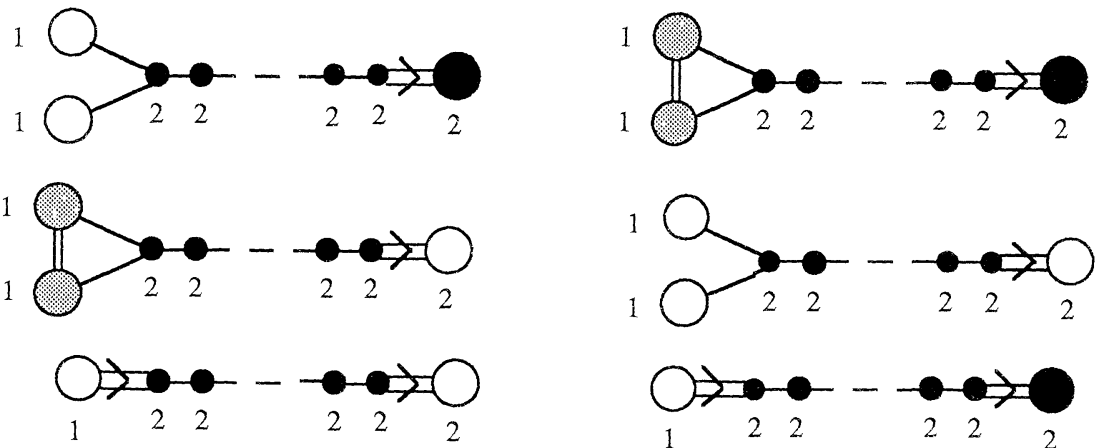

$$
\mathrm{K}=1
$$

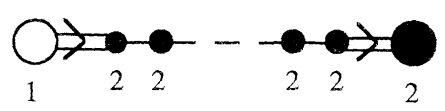

$\mathrm{SL}(\mathrm{m} / \mathrm{n})$

(1)

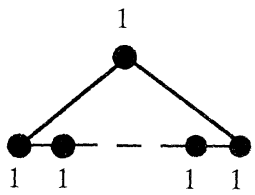


Table 9 (continued)

$$
\mathrm{G}(3)^{(1)}
$$
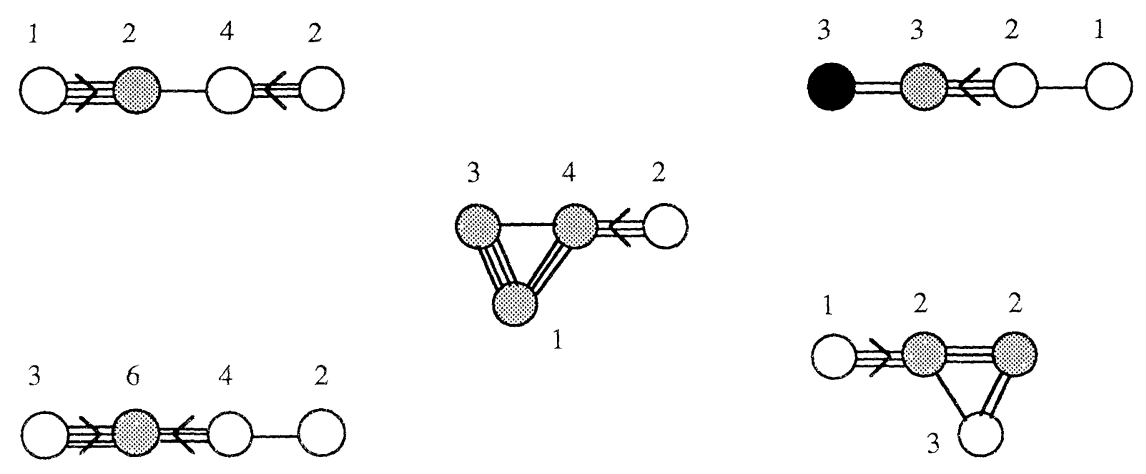

$F(4){ }^{(1)}$
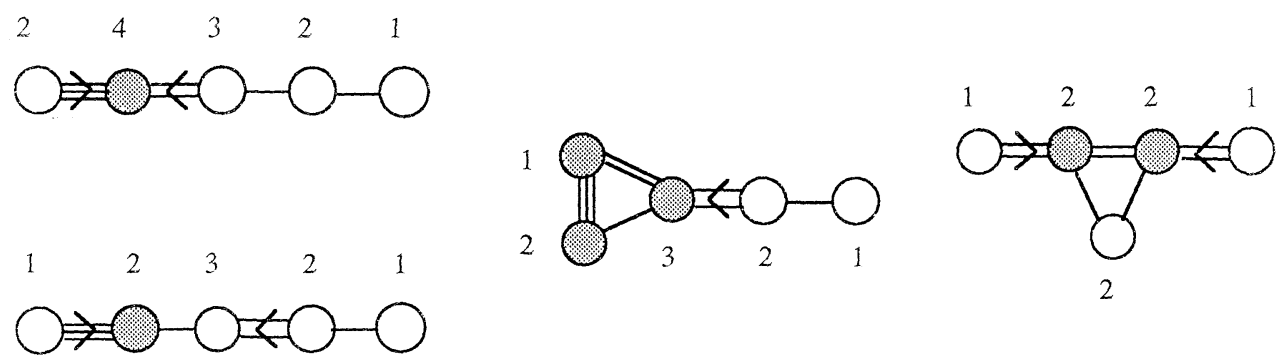

$$
\mathrm{D}(2,1 ; \alpha)^{(1)}
$$
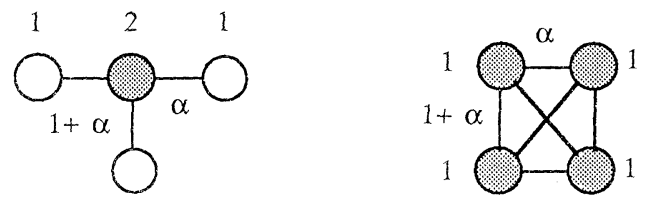
(super)algebra $\hat{\mathscr{G}}_{0}$ of $\mathscr{G}$ and a $\hat{\mathscr{G}}_{0}$-representation $\hat{\mathscr{G}}_{1}$ of this algebra, $\hat{\mathscr{G}}_{0}$ and $\hat{\mathscr{G}}_{1}$ satisfying Eq. (5.7). A Dynkin diagram of $\mathscr{G}^{(2)}$ is obtained by adding to the Dynkin diagram of $\hat{\mathscr{G}}_{0}$ the lowest weight of the $\hat{\mathscr{G}}_{0}$-representation $\hat{\mathscr{G}}_{1}$. We will call such a diagram a distinguished one. All the possible diagrams are obtained then by using the transformation (2.20) with respect to the grey dots. Let us see in detail how it works for the two kinds of twisted B.S.A., namely $S l(m \mid n)^{(2)}$ and $\operatorname{SSp}(2 m \mid 2 n)^{(2)}$.

For the $S l(m \mid n)^{(2)}$ case (with $m, n \geqq 3$ ), one can choose $\hat{\tau}$ such that the invariant subalgebra $\hat{\mathscr{G}}_{0}$ is $O(m) \oplus O(n)$ and the $\hat{\mathscr{G}}_{0}$-representation is the $(m, n)$ representation of $O(m) \oplus O(n)$. A simple root system of the invariant part is given by

$$
\begin{array}{ll}
\Delta=\left\{\varepsilon_{1}-\varepsilon_{2}, \ldots, \varepsilon_{m-1}-\varepsilon_{m}, \varepsilon_{m-1}+\varepsilon_{m}\right\} & \text { for } O(2 m), \\
\Delta=\left\{\varepsilon_{1}-\varepsilon_{2}, \ldots, \varepsilon_{m-1}-\varepsilon_{m}, \varepsilon_{m}\right\} & \text { for } O(2 m+1) \text { and } \\
\Delta=\left\{\delta_{1}-\delta_{2}, \ldots, \delta_{n-1}-\delta_{n}, \delta_{n-1}+\delta_{n}\right\} & \text { for } O(2 n), \\
\Delta=\left\{\delta_{1}-\delta_{2}, \ldots, \delta_{n-1}-\delta_{n}, \delta_{n}\right\} & \text { for } O(2 n+1)
\end{array}
$$

The lowest weight of the representation $(m, n)$ is $-\varepsilon_{1}-\delta_{1}$.

One has therefore a Dynkin diagram of the type

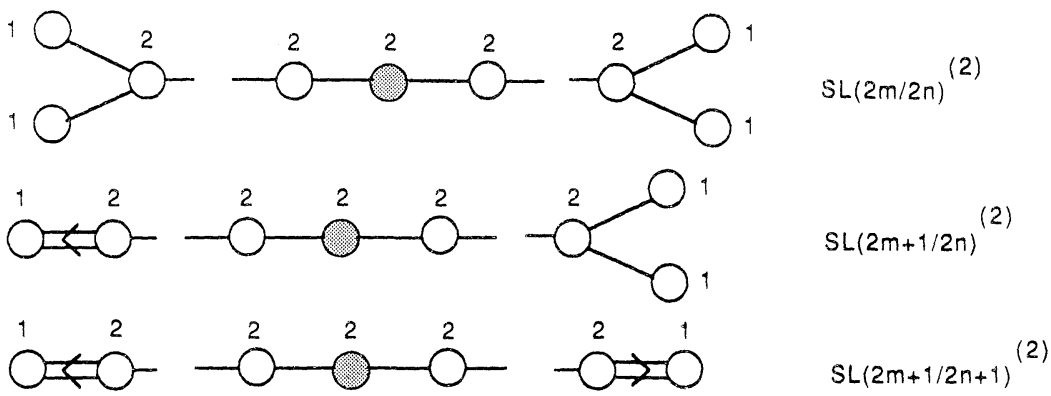

In the case $S l(2 \mid 2 n)^{(2)}$ (with $\left.n \geqq 2\right)$ (respectively $S l(2 \mid 2 n+1)^{(2)}$ (with $\left.n \geqq 1\right)$ ), the automorphism $\hat{\tau}$ can be taken such that the invariant subsuperalgebra $\hat{\mathscr{G}}_{0}$ is $O S p(2 n \mid 2)$ (respectively $O S p(2 n+1 \mid 2))$. The $\hat{\mathscr{G}}_{0}$-representation $\hat{\mathscr{G}}_{1}$ is the $(2 n, 2)$ (respectively $(2 n+1,2))$ representation of $S O(2 n) \otimes S p(2)$ (respectively $S O(2 n+1) \otimes S p(2))$, whose lowest weight is $-\varepsilon-\delta_{1}$. The distinguished simple root system associated to the distinguished diagram of the invariant part $O S p(2 n \mid 2)$ (respectively $O S p(2 n+1 \mid 2))$ is

$$
\begin{array}{lll}
\Delta=\left\{\varepsilon-\delta_{1}, \delta_{1}-\delta_{2}, \ldots, \delta_{n-1}-\delta_{n}, \delta_{n-1}+\delta_{n}\right\} & \text { for } & \operatorname{OSp}(2 n \mid 2), \\
\Delta=\left\{\varepsilon-\delta_{1}, \delta_{1}-\delta_{2}, \ldots, \delta_{n-1}-\delta_{n}, \delta_{n}\right\} & \text { for } & \operatorname{OSp}(2 n+1 \mid 2) .
\end{array}
$$

Therefore the Dynkin diagrams for $S l(2 \mid 2 n)^{(2)}$ and $S l(2 \mid 2 n+1)^{(2)}$ are respectively

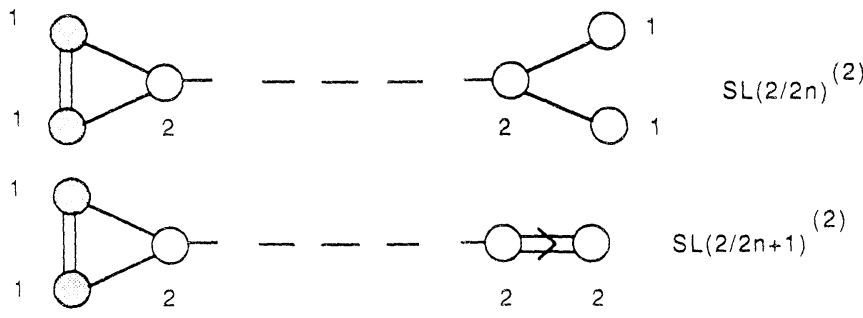


In the case of $S l(1 \mid 2 n)^{(2)}$ (with $n \geqq 2$ ), one can choose as invariant subalgebra $\hat{\mathscr{G}}_{0}$ $O(2 n)$. The $\hat{\mathscr{G}}_{0}$-representation $\hat{\mathscr{G}}_{1}$ is the fundamental representation $(2 n)$ of $O(2 n)$. Its lowest weight is $-\delta_{1}$. Therefore the Dynkin diagram of $\operatorname{Sl}(1 \mid 2 n)^{(2)}$ is

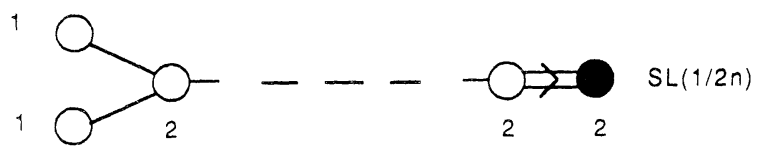

For the $O S p(2 m \mid 2 n)^{(2)}$ case (with $\left.m \geqq 2\right)$, one can choose $\hat{\tau}$ such that the invariant subsuperalgebra $\hat{\mathscr{G}}_{0}$ is $O \operatorname{Sp}(2 m-1 \mid 2 n)$. A simple root system of the invariant part is given by

$$
\Delta=\left\{\delta_{1}-\delta_{2}, \ldots, \delta_{n-1}-\delta_{n}, \delta_{n}-\varepsilon_{1}, \varepsilon_{1}-\varepsilon_{2}, \ldots, \varepsilon_{m-1}-\varepsilon_{m}, \varepsilon_{m}\right\} .
$$

The $\hat{\mathscr{G}}_{0}$-representation $\hat{\mathscr{G}}_{1}$ is the fundamental representation of $O \operatorname{Sp}(2 m-1 \mid 2 n)$ whose lowest weight is $-\delta_{1}$. The Dynkin diagram for $O \operatorname{Sp}(2 m \mid 2 n)^{(2)}$ is then
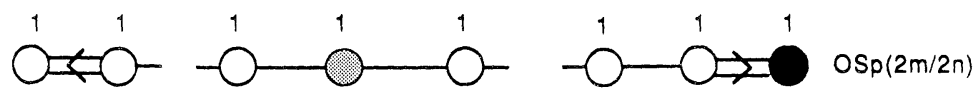

In the case of $\operatorname{OSp}(2 \mid 2 n)^{(2)}$, there exist an automorphism $\hat{\tau}$ such that the invariant subsuperalgebra $\hat{\mathscr{G}}_{0}$ is $O S p(1 \mid 2 n)$. The $\hat{\mathscr{G}}_{0}$-representation $\hat{\mathscr{G}}_{1}$ is the fundamental representation of $O S p(1 \mid 2 n)$. The simple root system of the invariant part $\hat{\mathscr{G}}_{0}$ is

$$
\Delta=\left\{\delta_{1}-\delta_{2}, \ldots, \delta_{n-1}-\delta_{n}, \delta_{n}\right\} .
$$

The lowest weight of the $\hat{\mathscr{G}}_{1}$ representation of $\hat{\mathscr{G}}_{0}$ is $-\delta_{1}$. One obtains the following Dynkin diagram for $\operatorname{OSp}(2 \mid 2 n)^{(2)}$ :

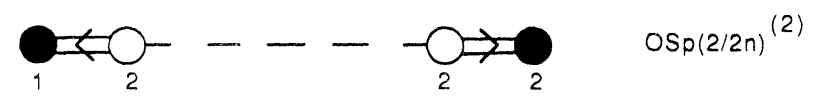

For $\operatorname{Sl}(1 \mid 2 n+1)^{(4)}$ the invariant subalgebra can be taken as $O(2 n+1)$ and the $\hat{\mathscr{G}}_{0}$ representation $\hat{\mathscr{G}}_{1}$ is the fundamental representation of $O(2 n+1)$ whose lowest weight is $-\delta_{1}$. The Dynkin diagram of $S l(1 \mid 2 n+1)^{(4)}$ is thus

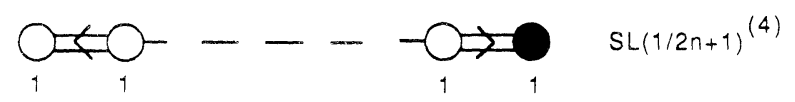

Some more details about this particular case can be found in [16].

The Dynkin diagrams for the twisted affine B.S.A. of rank less than 4 of the unitary and orthosymplectic series are constructed in Table 10, whereas the general form of all the twisted B.S.A. is given in Table 11.

\subsection{Folding Method for Affine B.S.A.}

The folding method (see Sect. 4) applied to Dynkin diagrams of affine Lie algebras can lead in certain conditions to twisted affine Lie algebras [17]. Such a property can also be obtained for affine B.S.A. Hereafter we will study the folding of symmetric Dynkin diagrams associated to affine B.S.A. Special inclusions among 
Table 10. Dynkin diagrams of twisted unitary and orthosymplectic superalgebras of rank 1,2,3,4

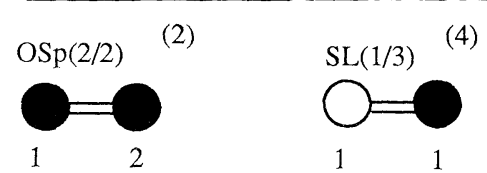

(2)

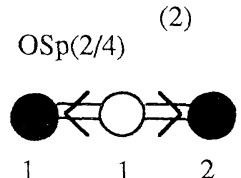

(2)

$\operatorname{OSp}(4 / 2)$
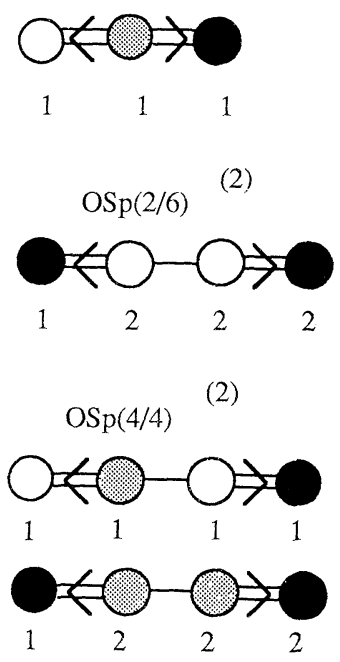

$\operatorname{OSp}(6 / 2)$
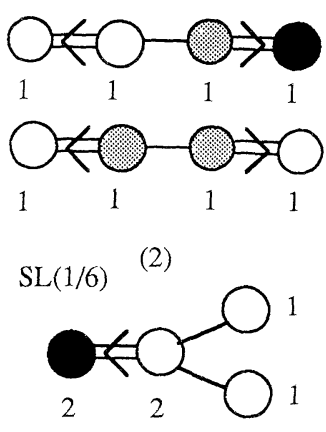

(2)

SL(1/4)

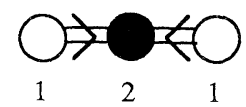

(2)
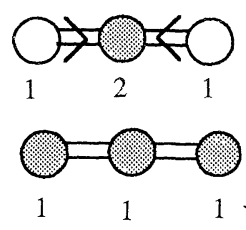

$\operatorname{SL}(2 / 4)$
$\operatorname{SL}(3 / 2) \quad(2)$
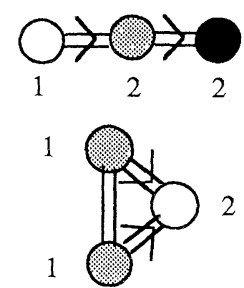

(2)
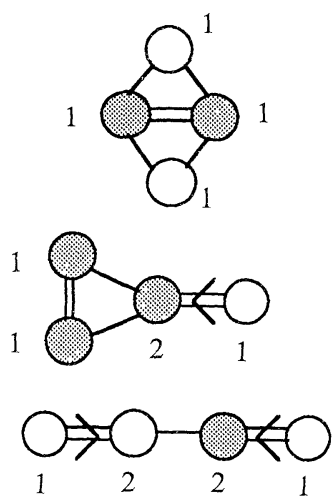

$\operatorname{SL}(5 / 2)$
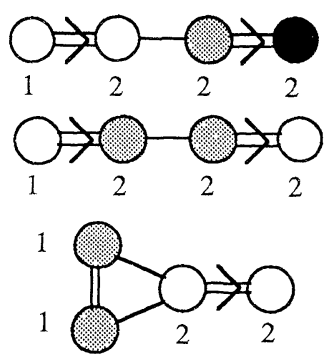

SL(1/5)

(4)

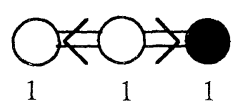

SL(3/3)

(2)
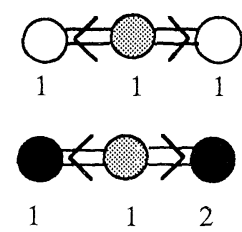

SL(3/4)

(2)
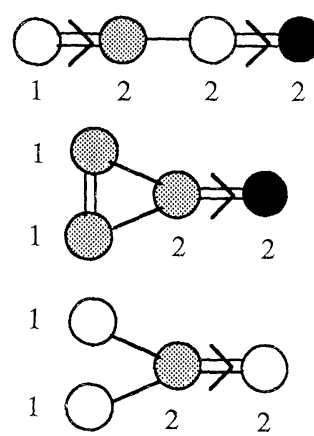

(2)

SL(3/5)
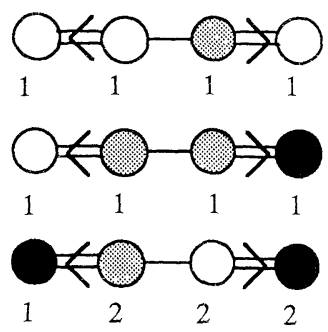

$\mathrm{SL}(1 / 7)$

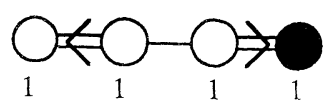


Table 11. Dynkin diagrams of twisted affine B.S.A

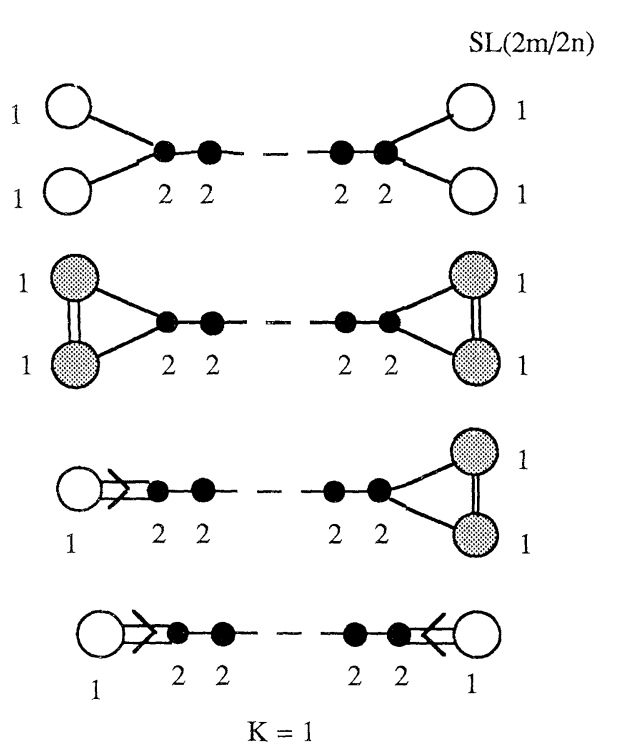

1
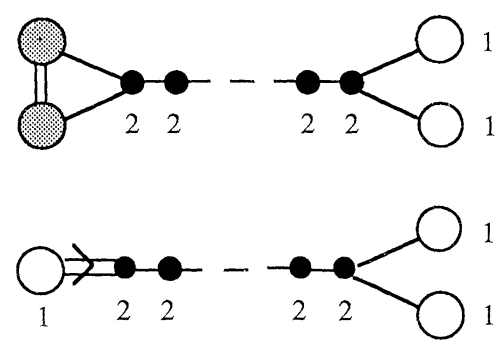

$\mathrm{K}=0$

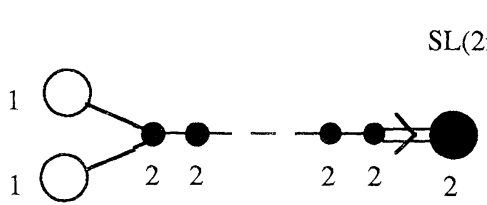

(2)
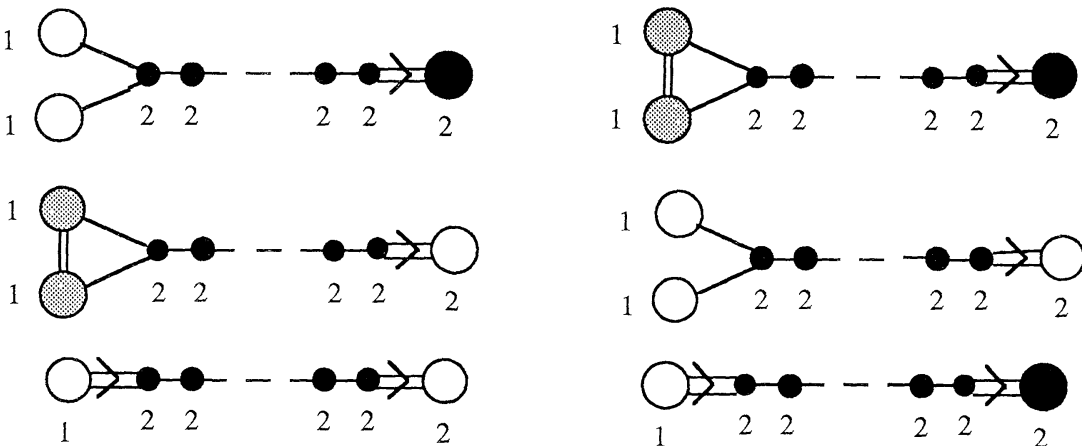

$\mathrm{K}=0$
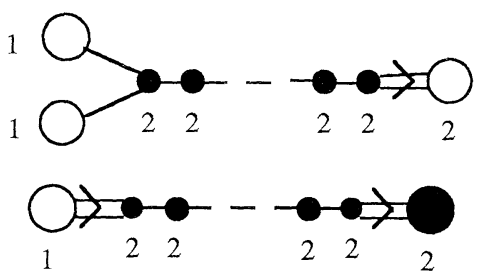

$$
\mathrm{K}=1
$$

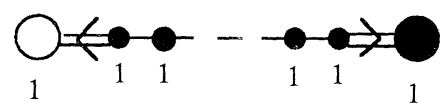

$$
\begin{array}{lll}
\operatorname{OSp}(2 \mathrm{~m} / 2 \mathrm{n}) & \mathrm{K}=1 \\
\operatorname{SL}(2 \mathrm{~m}+1 / 2 \mathrm{n}+1) & (2) & \mathrm{K}=0
\end{array}
$$

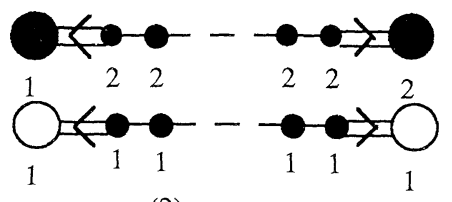

$\operatorname{OSp}(2 \mathrm{~m} / 2 \mathrm{n})$ $\operatorname{SL}(2 \mathrm{~m}+1 / 2 \mathrm{n}+1)$ 
affine B.S.A. will be obtained. More precisely we will see that starting from the set of untwisted affine B.S.A.'s, successive foldings allow us to reconstruct all the possible twisted B.S.A.

- Folding of $O \operatorname{Sp}(2 m \mid 2 n)^{(1)}$ (with $m \geqq 2$ )

Let us start with the following Dynkin diagram of $O \operatorname{Sp}(2 m \mid 2 n)^{(1)}$ which has a $Z_{2}$ symmetry

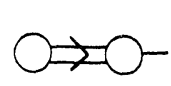

$\alpha_{0} \alpha_{1}$
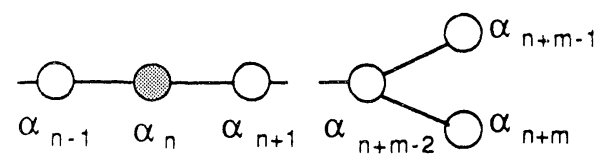

$\operatorname{OSp}(2 m / 2 n)$

The corresponding simple root system is

$$
\begin{gathered}
\Delta=\left\{\alpha_{0}=k-2 \delta_{1}, \alpha_{1}=\delta_{1}-\delta_{2}, \ldots, \alpha_{n-1}=\delta_{n-1}-\delta_{n}, \alpha_{n}=\delta_{n}-\varepsilon_{1},\right. \\
\left.\alpha_{n+1}=\varepsilon_{1}-\varepsilon_{2}, \ldots, \alpha_{n+m-1}=\varepsilon_{m-1}-\varepsilon_{m}, \alpha_{n+m}=\varepsilon_{m-1}+\varepsilon_{m}\right\},
\end{gathered}
$$

where $k$ is the isotropic affine direction. The folding is defined by the automorphism $\tau$ such that

$$
\tau\left(\alpha_{i}\right)=\alpha_{i}(i=0, \ldots, m+n-2) \quad \tau\left(\alpha_{n+m-1}\right)=\alpha_{n+m} \quad \tau^{2}=1 .
$$

The folded Dynkin diagram is

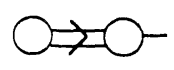

$\alpha_{0} \alpha_{1}$

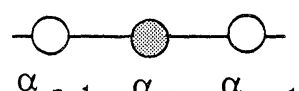

$\alpha_{n-1} \alpha_{n} \alpha_{n+1}$

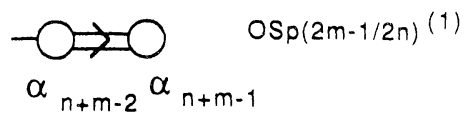

with $\alpha_{n+m-1}^{\prime}=\left(\alpha_{n+m-1}+\alpha_{n+m}\right) / 2$ and the corresponding folded simple root system is

$$
\begin{aligned}
\Delta^{\prime}= & \left\{\alpha_{0}=k-2 \delta_{1}, \alpha_{1}=\delta_{1}-\delta_{2}, \ldots, \alpha_{n-1}=\delta_{n-1}-\delta_{n}, \alpha_{n}=\delta_{n}-\varepsilon_{1},\right. \\
& \left.\alpha_{n+1}=\varepsilon_{1}-\varepsilon_{2}, \ldots, \alpha_{n+m-1}^{\prime}=\varepsilon_{m-1}\right\} .
\end{aligned}
$$

Therefore, the folding of the affine superalgebra $\operatorname{OSp}(2 m \mid 2 n)^{(1)}$ leads to the superalgebra $O S p(2 m-1 \mid 2 n)^{(1)}$. See the general schemes in Table 12.

- Folding of $\operatorname{OSp}(2 \mid 2 n)^{(1)}$

In the case of $O \operatorname{Sp}(2 \mid 2 n)^{(1)}$, the $Z_{2}$ symmetric diagram is

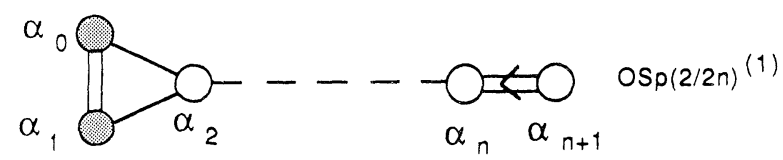

with the simple root system

$\Delta=\left\{\alpha_{0}=k-\varepsilon-\delta_{1}, \alpha_{1}=\varepsilon-\delta_{1}, \alpha_{2}=\delta_{1}-\delta_{2}, \ldots, \alpha_{n}=\delta_{n-1}-\delta_{n}, \alpha_{n+1}=2 \delta_{n}\right\}$.

The folding is defined by

$$
\tau\left(\alpha_{i}\right)=\alpha_{i}(i=2, \ldots, n+1) \quad \tau\left(\alpha_{0}\right)=\alpha_{1} \quad \tau^{2}=1 .
$$


Table 12. Folding schemes for affine B.S.A.

$\operatorname{OSp}(2 \mathrm{~m} / 2 \mathrm{n})$

(1)

$\operatorname{OSp}(2 m-1 / 2 n)$

(1)

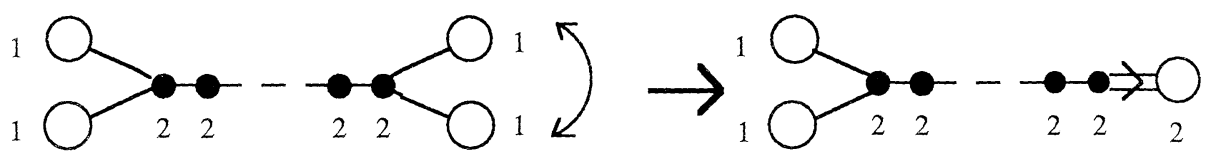

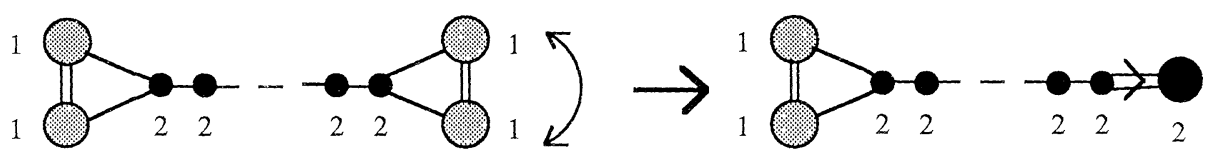

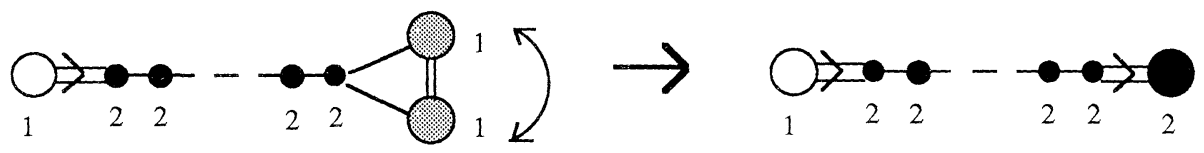

$\mathrm{K}=0$

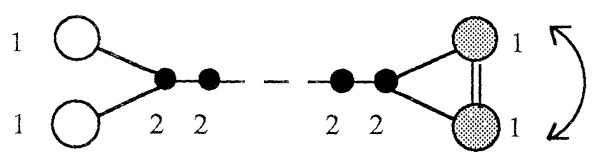

$\rightarrow \overbrace{2}-\infty-\infty$

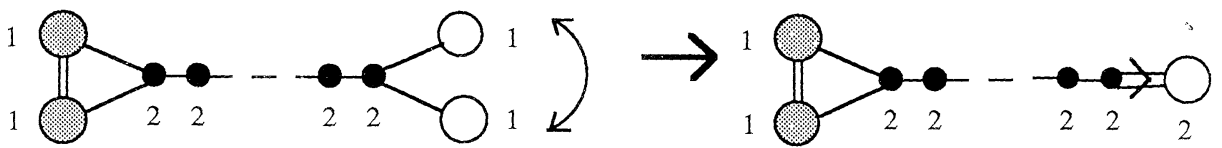

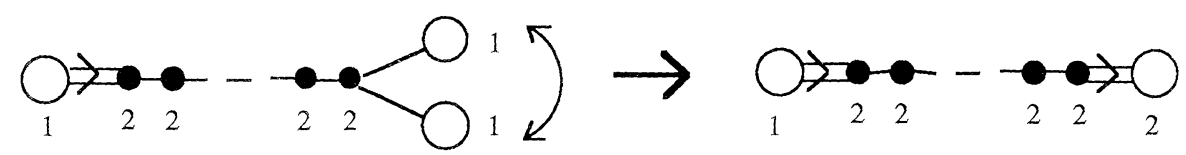

$\mathrm{K}=1$ 
Table 12 (continued)

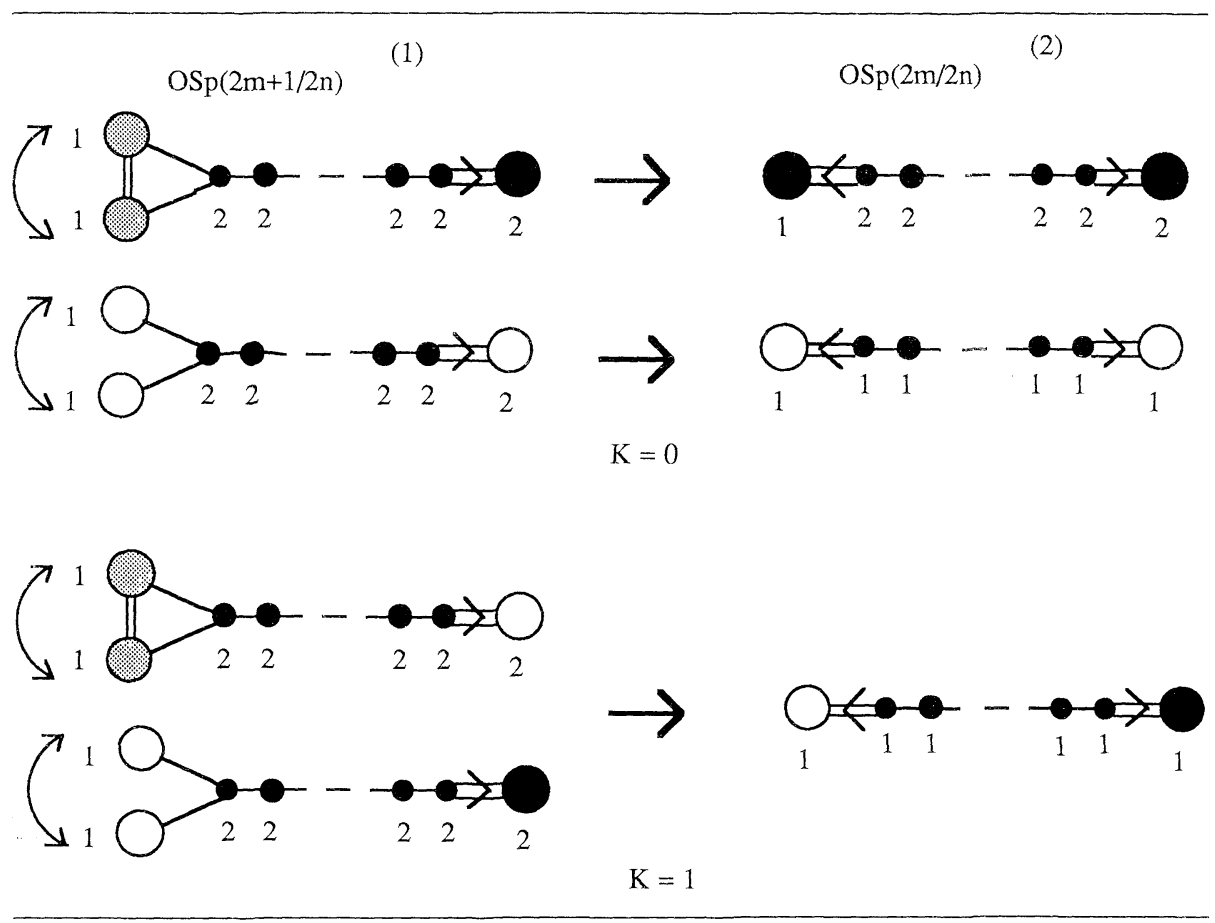

The folded Dynkin diagram is

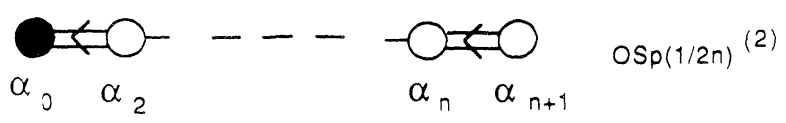

corresponding to the simple root system

$$
\Delta^{\prime}=\left\{\alpha_{0}^{\prime}=k / 2-\delta_{1}, \alpha_{2}=\delta_{1}-\delta_{2}, \ldots, \alpha_{n}=\delta_{n-1}-\delta_{n}, \alpha_{n+1}=2 \delta_{n}\right\}
$$

with $\alpha_{0}^{\prime}=\left(\alpha_{0}+\alpha_{1}\right) / 2$. One obtains the twisted superalgebra $O \operatorname{Sp}(1 \mid 2 n)^{(2)}$ which is actually isomorphic to $O \operatorname{Sp}(1 \mid 2 n)^{(1)}$.

- Folding of $O S p(2 m+1 \mid 2 n)^{(1)}$ (with $m \geqq 2$ )

We consider the following $Z_{2}$ symmetric Dynkin diagram of $O S p(2 m+1 \mid 2 n)^{(1)}$

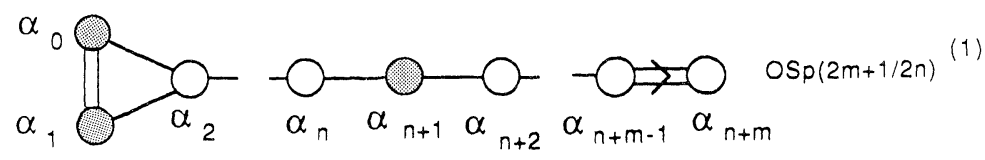

with its simple root system

$$
\begin{aligned}
\Delta=\left\{\alpha_{0}=k-\delta_{1}-\varepsilon_{1}, \alpha_{1}=\varepsilon_{1}-\delta_{1}, \alpha_{2}=\delta_{1}-\delta_{2}, \ldots, \alpha_{n}=\delta_{n-1}-\delta_{n},\right. \\
\\
\left.\alpha_{n+1}=\delta_{n}-\varepsilon_{2}, \alpha_{n+2}=\varepsilon_{2}-\varepsilon_{3}, \ldots, \alpha_{n+m-1}=\varepsilon_{m-1}-\varepsilon_{m}, \alpha_{n+m}=\varepsilon_{m}\right\} .
\end{aligned}
$$


The folding is defined by the automorphism $\tau$

$$
\tau\left(\alpha_{i}\right)=\alpha_{i}(i=2, \ldots, n+m) \quad \tau\left(\alpha_{0}\right)=\alpha_{1} \quad \tau^{2}=1 .
$$

The folded Dynkin diagram is
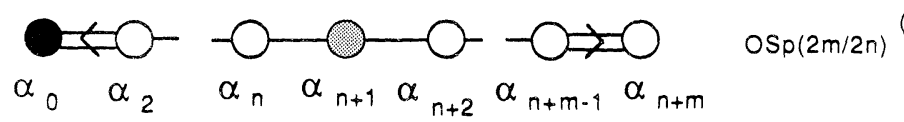

and the corresponding simple root system is

$$
\begin{gathered}
\Delta^{\prime}=\left\{\alpha_{0}^{\prime}=k / 2-\delta_{1}, \alpha_{2}=\delta_{1}-\delta_{2}, \ldots, \alpha_{n}=\delta_{n-1}-\delta_{n}, \alpha_{n+1}=\delta_{n}-\varepsilon_{2},\right. \\
\left.\alpha_{n+2}=\varepsilon_{2}-\varepsilon_{3}, \ldots, \alpha_{n+m-1}=\varepsilon_{m-1}-\varepsilon_{m}, \alpha_{n+m}=\varepsilon_{m}\right\}
\end{gathered}
$$

with $\alpha_{0}^{\prime}=\left(\alpha_{0}+\alpha_{1}\right) / 2$. One obtains as folded superalgebra the twisted affine superalgebra $O S p(2 m \mid 2 n)^{(2)}$. See also the general schemes in Table 12.

- Folding of $O \operatorname{Sp}(3 \mid 2 n)^{(1)}$

In the case of $O S p(3 \mid 2 n)^{(1)}$, the $Z_{2}$ symmetric diagram is

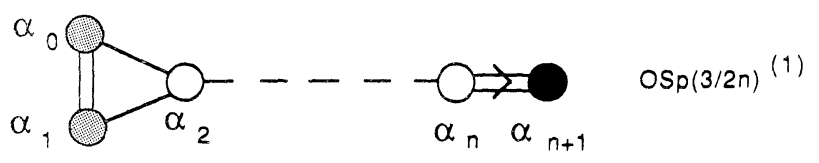

with the simple root system

$\Delta=\left\{\alpha_{0}=k-\varepsilon-\delta_{1}, \alpha_{1}=\varepsilon-\delta_{1}, \alpha_{2}=\delta_{1}-\delta_{2}, \ldots, \alpha_{n}=\delta_{n-1}-\delta_{n}, \alpha_{n+1}=\delta_{n}\right\}$.

The folding is defined by

$$
\tau\left(\alpha_{i}\right)=\alpha_{i}(i=2, \ldots, n+1) \quad \tau\left(\alpha_{0}\right)=\alpha_{1} \quad \tau^{2}=1 .
$$

The folded Dynkin diagram is

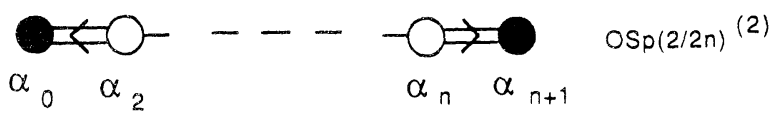

corresponding to the simple root system

$$
\Delta^{\prime}=\left\{\alpha_{0}^{\prime}=k / 2-\delta_{1}, \alpha_{2}=\delta_{1}-\delta_{2}, \ldots, \alpha_{n}=\delta_{n-1}-\delta_{n}, \alpha_{n+1}=\delta_{n}\right\}
$$

with $\alpha_{0}^{\prime}=\left(\alpha_{0}+\alpha_{1}\right) / 2$. One obtains the twisted superalgebra $\operatorname{OSp}(2 \mid 2 n)^{(2)}$.

- Folding of $S l(2 m \mid 2 n)^{(1)}$ (with $m, n \geqq 2$ )

One starts from the distinguished diagram of $S l(2 m \mid 2 n)^{(1)}$ (which is simply the extended diagram of the distinguished diagram of $S l(2 m \mid 2 n)$ ),

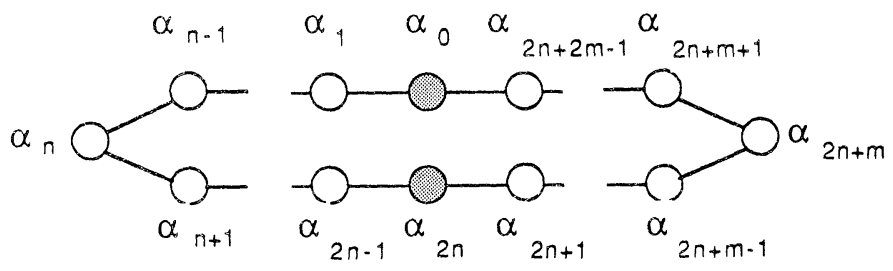


The associated simple root system is

$$
\begin{aligned}
\Delta=\left\{\alpha_{0}=k-\delta_{1}+\varepsilon_{2 m}, \alpha_{1}\right. & =\delta_{1}-\delta_{2}, \ldots, \alpha_{2 n-1}=\delta_{2 n-1}-\delta_{2 n}, \\
\alpha_{2 n}=\delta_{2 n}-\varepsilon_{1}, \alpha_{2 n+1} & \left.=\varepsilon_{1}-\varepsilon_{2}, \ldots, \alpha_{2 n+2 m-1}=\varepsilon_{2 m-1}-\varepsilon_{2 m}\right\} .
\end{aligned}
$$

The folding is defined by the automorphism $\tau$ of order 2 such that

$$
\tau\left(\alpha_{i}\right)=\alpha_{2 n-i}(i=0, \ldots, 2 n) \quad \tau\left(\alpha_{j}\right)=\alpha_{4 n+2 m-j}(j=2 n+1, \ldots, 2 n+2 m-1) .
$$

The folded Dynkin diagram is
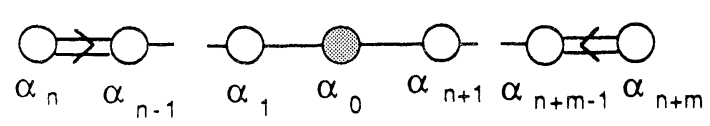

$S L(2 m / 2 n)$

and the simple root system attached to it is

$$
\begin{aligned}
\Delta^{\prime}= & \left\{\alpha_{0}^{\prime}=k / 2-\Delta_{1}-E_{1}, \alpha_{1}^{\prime}=\Delta_{1}-\Delta_{2}, \ldots, \alpha_{n-1}^{\prime}=\Delta_{n-1}-\Delta_{n},\right. \\
& \left.\alpha_{n}^{\prime}=2 \Delta_{n}, \alpha_{n+1}^{\prime}=E_{1}-E_{2}, \ldots, \alpha_{n+m-1}^{\prime}=E_{m-1}-E_{m}, \alpha_{n+m}^{\prime}=2 E_{m}\right\},
\end{aligned}
$$

where $E_{i}$ and $\Delta_{i}$ correspond to the rescaled roots

$$
E_{i}=\left(\varepsilon_{i}-\varepsilon_{2 m+1-i}\right) / 2(i=1, \ldots, m) \quad \Delta_{j}=\left(\delta_{j}-\delta_{2 n+1-j}\right) / 2(j=1, \ldots, n)
$$

with $\alpha_{i}^{\prime}=\left(\alpha_{i}+\alpha_{2 n-1}\right) / 2(i=1, \ldots, n)$ and $\alpha_{j-n}^{\prime}=\left(\alpha_{j}+\alpha_{4 n+2 m-j}\right) / 2(j=2 n+1, \ldots$, $2 n+m)$. One obtains finally the twisted affine superalgebra $\operatorname{Sl}(2 m \mid 2 n)^{(2)}$.

- Folding of $S l(2 m \mid 2 n)^{(2)}$ (with $m, n \geqq 2$ )

Consider the Dynkin diagram of $S l(2 m \mid 2 n)^{(2)}$ obtained just above. At first sight, this diagram has no apparent symmetry. Using Weyl transformations with respect to grey dots, we will transform this diagram into a $Z_{2}$ symmetric one, as follows:
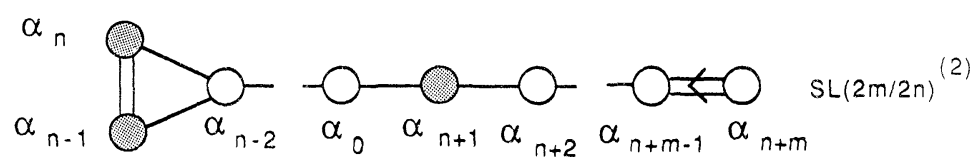

which corresponds to the simple root system

$$
\begin{aligned}
\Delta=\{ & \alpha_{0}=k / 2+\delta_{1}-\delta_{2}, \alpha_{1}=\delta_{2}-\delta_{3}, \ldots, \alpha_{n-2}=\delta_{n-1}-\delta_{n}, \\
& \alpha_{n-1}=\delta_{n}+\varepsilon_{1}, \alpha_{n}=\delta_{n}-\varepsilon_{1}, \alpha_{n+1}=-\delta_{2}-\varepsilon_{2}, \alpha_{n+2}=\varepsilon_{2}-\varepsilon_{3}, \ldots, \\
& \left.\alpha_{n+m-1}=\varepsilon_{m-1}-\varepsilon_{m}, \alpha_{n+m}=2 \varepsilon_{m}\right\} .
\end{aligned}
$$

The automorphism $\tau$ associated to the folding is

$$
\tau\left(\alpha_{i}\right)=\alpha_{i}(i=1, \ldots, n+m, i \neq n-1, n) \quad \tau\left(\alpha_{n-1}\right)=\alpha_{n} \quad \tau^{2}=1 .
$$

The folded Dynkin diagram is

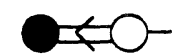

$\alpha_{n-1} \alpha_{n-2}$

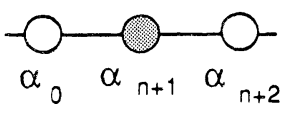

$\alpha_{n+2}$

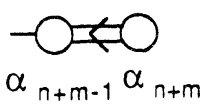

$S L(2 m-1 / 2 n)^{(2)}$ 
with the simple root system

$$
\begin{aligned}
\Delta^{\prime}== & \left\{\alpha_{0}=k / 2+\delta_{1}-\delta_{2}, \alpha_{1}=\delta_{2}-\delta_{3}, \ldots, \alpha_{n-2}=\delta_{n-1}-\delta_{n}, \alpha_{n-1}^{\prime}=\delta_{n},\right. \\
& \left.\alpha_{n+1}=-\delta_{2}-\varepsilon_{2}, \alpha_{n+2}=\varepsilon_{2}-\varepsilon_{3}, \ldots, \alpha_{n+m-1}=\varepsilon_{m-1}-\varepsilon_{m}, \alpha_{n+m}=2 \varepsilon_{m}\right\}
\end{aligned}
$$

with $\alpha_{n-1}^{\prime}=\left(\alpha_{n-1}+\alpha_{n}\right) / 2$. The folding of the $\operatorname{Sl}(2 m \mid 2 n)^{(2)}$ superalgebra leads therefore to the $\operatorname{Sl}(2 m-1 \mid 2 n)^{(2)}$ superalgebra.

- Folding of $S l(2 m-1 \mid 2 n)^{(2)}$ (with $m, n \geqq 2$ )

Let us start with the previous diagram of $S l(2 m-1 \mid 2 n)^{(2)}$. After Weyl transformations with respect to the grey dot, we obtain the following diagram, which exhibits a $Z_{2}$ symmetry

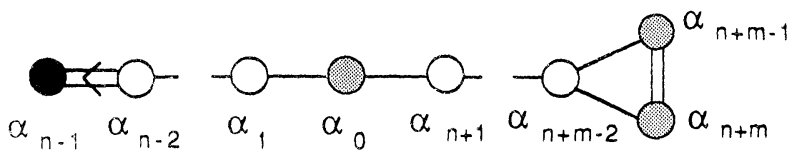

The associated root system is

$$
\begin{aligned}
\Delta= & \left\{\alpha_{0}=k / 2+\varepsilon_{2}-\delta_{2}, \alpha_{1}=\delta_{2}-\delta_{3}, \ldots, \alpha_{n-2}=\delta_{n-1}-\delta_{n}, \alpha_{n-1}=\delta_{n},\right. \\
& \left.\alpha_{n+1}=\varepsilon_{2}-\varepsilon_{3}, \ldots, \alpha_{n+m-2}=\varepsilon_{m-1}-\varepsilon_{m}, \alpha_{n+m-1}=\varepsilon_{m}+\delta_{1}, \alpha_{n+m}=\varepsilon_{m}-\delta_{1}\right\} .
\end{aligned}
$$

The automorphism $\tau$ defining the folding is

$$
\tau\left(\alpha_{i}\right)=\alpha_{i}(i=1, \ldots, n+m-2) \quad \tau\left(\alpha_{n+m-1}\right)=\alpha_{n+m} \quad \tau^{2}=1 .
$$

The folded Dynkin diagram is

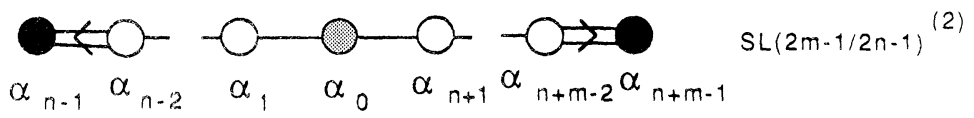

with the simple root system

$$
\begin{gathered}
\Delta^{\prime}=\left\{\alpha_{0}=k / 2+\varepsilon_{2}-\delta_{2}, \alpha_{1}=\delta_{2}-\delta_{3}, \ldots, \alpha_{n-2}=\delta_{n-1}-\delta_{n}, \alpha_{n-1}=\delta_{n},\right. \\
\left.\alpha_{n+1}=\varepsilon_{2}-\varepsilon_{3}, \ldots, \alpha_{n+m-2}=\varepsilon_{m-1}-\varepsilon_{m}, \alpha_{n+m-1}^{\prime}=\varepsilon_{m}\right\},
\end{gathered}
$$

with $\alpha_{n+m-1}^{\prime}=\left(\alpha_{n+m-1}+\alpha_{n+m}\right) / 2$. The folding of $S l(2 m-1 \mid 2 n)^{(2)}$ gives the twisted superalgebra $\operatorname{Sl}(2 m-1 \mid 2 n-1)^{(2)}$.

- Folding of $S l(2 \mid 2 n)^{(2)}$ and $S l(1 \mid 2 n)^{(2)}(n \geqq 2)$

The case of $S l(2 \mid 2 n)^{(2)}$ and $S l(1 \mid 2 n)^{(2)}$ demands special attention since the resulting folded diagram doesn't contain any grey dot, i.e. any fermionic root of null length. Consider the diagram of $S /(2 \mid 2 n)^{(2)}$ which is constructed by the standard way explained above

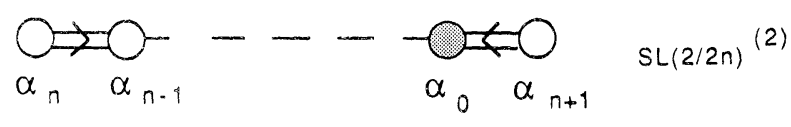


Successive Weyl transformations with respect to the grey dots lead to the diagram

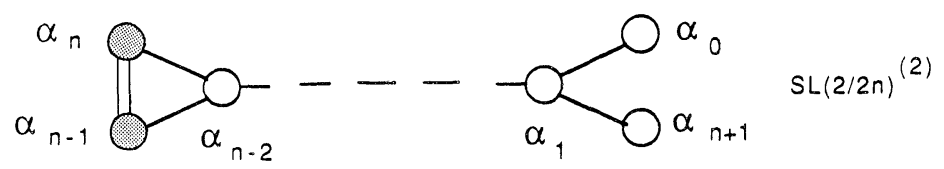

with the simple root system

$$
\begin{gathered}
\Delta=\left\{\alpha_{0}=k / 2-\delta_{1}-\delta_{2}, \alpha_{1}=\delta_{2}-\delta_{3}, \ldots, \alpha_{n-2}=\delta_{n-1}-\delta_{n},\right. \\
\left.\alpha_{n-1}=\delta_{n}-\varepsilon, \alpha_{n}=\delta_{n}+\varepsilon, \alpha_{n+1}=\delta_{1}-\delta_{2}\right\} .
\end{gathered}
$$

The $Z_{2}$ symmetry of the left of the diagram is associated to the automorphism $\tau$ such that

$$
\tau\left(\alpha_{i}\right)=\alpha_{i}(i=0, \ldots, n-2) \quad \tau\left(\alpha_{n-1}\right)=\alpha_{n} \quad \tau^{2}=1 .
$$

The folded Dynkin diagram is

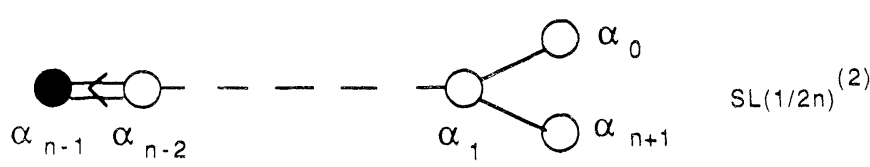

and the folded simple root system is

$$
\begin{aligned}
& \quad \Delta^{\prime}=\left\{\alpha_{0}=k / 2-\delta_{1}-\delta_{2}, \alpha_{1}=\delta_{2}-\delta_{3}, \ldots, \alpha_{n-2}=\delta_{n-1}-\delta_{n},\right. \\
& \left.\alpha_{n-1}^{\prime}=\delta_{n}, \alpha_{n+1}=\delta_{1}-\delta_{2}\right\} \\
& \text { with } \alpha_{n-1}^{\prime}=\left(\alpha_{n+1}+\alpha_{n}\right)^{2},
\end{aligned}
$$

which corresponds to the twisted superalgebra $S l(1 \mid 2 n)^{(2)}$.

Now the remaining $Z_{2}$ symmetry of the diagram gives rise to the folding defined by

$$
\tau\left(\alpha_{i}\right)=\alpha_{i}(i=1, \ldots, n-1) \quad \tau\left(\alpha_{0}\right)=\alpha_{n+1} \quad \tau^{2}=1
$$

The folded diagram is

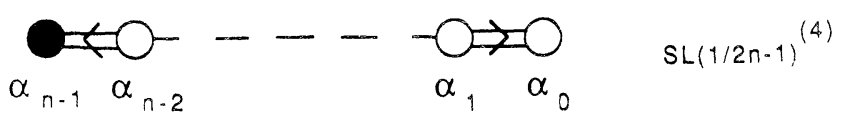

and the corresponding simple root system is

$$
\Delta^{\prime \prime}=\left\{\alpha_{0}^{\prime}=k / 4-\delta_{2}, \alpha_{1}=\delta_{2}-\delta_{3}, \ldots, \alpha_{n-2}=\delta_{n-1}-\delta_{n}, \alpha_{n-1}^{\prime}=\delta_{n}\right\}
$$

with $\alpha_{0}^{\prime}=\left(\alpha_{0}+\alpha_{n+1}\right) / 2$. One obtains therefore the twisted superalgebra $\operatorname{Sl}(1 \mid 2 n-1)^{(4)}$.

\section{Conclusion}

We have shown that the use of Dynkin diagrams, which is so convenient to deduce many characteristics of a Lie algebra, can be extended to the case of Lie 
superalgebras. The main difference between a simple Lie algebra and a superalgebra at this level is the possibility for a Lie superalgebra to admit several Dynkin diagrams. This technical difficulty is in part softened by a systematic method of determining the non-equivalent Dynkin diagrams [5]. It has therefore been possible to construct the regular sub(super)algebras of a B.S.A. and to recognize in most cases the outer automorphisms of a superalgebra by looking at the symmetries of its Dynkin diagrams. If some singular sub(super)algebras have been determined by folding symmetric diagrams, the general classification of singular sub(super)algebras of a B.S.A. is not yet known. Let us mention at this point a first step in that direction can be found in [18] where symmetric superspaces are studied - see also [19]. It is obvious to say that superalgebras constitute a natural ingredient in supersymmetric theories. Note in particular that in conformal extended supergravity the symmetry group is $S U(2,2 \mid N)$, which contains the (anti) De Sitter supergravity symmetry group $O S p(N \mid 4)$ [20] (see also Sect.4). In the spirit of a systematic research of possible symmetries in supergravity theories, the complete knowledge of sub(super)algebras of a unitary or orthosymplectic superalgebra might be useful. Among other relevant approaches, let us also note the attempt of treating supergravity models as theories of spontaneous breakdown in analogy to the pure Einstein gravity [21].

Still in the context of finite dimensional superalgebras, one may wonder to what extent the feature of a superalgebra to admit in general more than one Dynkin diagram can be exploited in the study of highest weight representations and also in the reduction of product of representations.

In the case of affine Lie superalgebras, symmetries of the Dynkin diagrams involving the affine root give a simple method for obtaining the twisted affine superalgebras. Vertex operators representation of orthosymplectic superalgebras have already been constructed [22]. Using special inclusions among superalgebras the case of $S U(m \mid n)$ vertex operator representation can also be studied, and the just mentioned folding procedure used to determine the vertex operator construction for twisted affine superalgebras [23]. Actually affine superalgebras show up when considering symplectic bosons appearing in the BRST treatment of fermionic string theories [24]: in this approach vertex operators occur in constructing the corresponding superconformal ghosts [25].

\section{References}

1. Kac, V.G.: Adv. Math. 26, 8 (1977)

2. Kac, V.G.: Lecture Notes in Math., Vol. 676, p. 597. Berlin, Heidelberg, New York: Springer 1978

3. Kac, V.G.: Commun. Math. Phys. 53, 31 (1977)

4. Serganova, V.V.: Math. USSR Izv. 24, 539 (1985)

5. Leites, D.A., Saveliev, M.V., Serganova, V.V.: Serpukhov preprint 85-81 (1985)

6. Van der Leur, J.W.: Contragredient Lie superalgebras of finite growth, Utrecht thesis (1985)

7. Dynkin, E. B.: Am. Math. Soc. Transl. 6, 111 (1957)

8. Lorente, M., Gruber, B.: J. Math. Phys. 13, 1639 (1972)

9. Van der Jeugt, J.: J. Math. Phys. 28, 292 (1987)

10. Helgason, S.: Differential geometry, Lie groups and symmetric spaces. New York: Academic Press 1978

11. Parker, M.: J. Math. Phys. 21, 689 (1980) 
12. Serganova, V.V.: Funct. Anal. Appl. 17, 200 (1983) and references therein

13. Goddard, P., Nahm, W., Olive, D.I., Schwimmer, A.: Commun. Math. Phys. 107, 179 (1986)

14. Kac, V.G.: Adv. Math. 30, 85 (1978)

15. Kac, V.G.: Infinite dimensional Lie algebras. Cambridge: Cambridge University Press 1985

16. Feingold, A., Frenkel, I. B.: Adv. Math. 56, 117 (1985)

17. Frappat, L., Sciarrino, A., Sorba, P.: Nucl. Phys. B 305 [FS 23], 164 (1988)

18. Serganova, V.V.: Funct. Anal. Appl. 17, 200 (1983) (English translation)

19. Leites, D. A.: Theory of supermanifolds, (in Russian). Izd. Karel. Fil. Akad. Nauk SSSR, Petrozavodsk, 1983

20. Ferrara, S., Kaku, M., Townsend, P.K., Van Nieuwenhuizen, P.: Nucl. Phys. B 129, 125 (1977)

21. Ivanov, E.A., Niederle, J.: Class. Quantum Grav. 2, 63 (1985)

22. Frappat, L.: Int. J. Mod. Phys. A 3, 2545 (1988)

23. Frappat, L., Sciarrino, A., Sorba, P.: LAPP TH 216/88

24. Goddard, P., Olive, D. I., Waterson, G.: Commun. Math. Phys. 112, 591 (1987)

25. Friedan, D., Martinec, E., Shenker, S.: Nucl. Phys. B 271, 93 (1986)

Communicated by L. Alvarez-Gaumé

Received February 23, 1988; in revised form June 14, 1988 